\title{
Oral administration of a recombinant cholera toxin B subunit promotes mucosal healing in the colon
}

\author{
KJ Baldauf ${ }^{1,5}$, JM Royal ${ }^{1,2,5}$, JC Kouokam², B Haribabu ${ }^{3}$, VR Jala ${ }^{3}$, K Yaddanapudi ${ }^{4}$, KT Hamorsky ${ }^{2,4}$, \\ GW Dryden ${ }^{4}$ and N Matoba ${ }^{1,2}$
}

Cholera toxin B subunit (CTB) is a component of a licensed oral cholera vaccine. However, CTB has pleiotropic immunomodulatory effects whose impacts on the gut are not fully understood. Here, we found that oral administration in mice of a plant-made recombinant CTB (СTBp) significantly increased several immune cell populations in the colon lamina propria. Global gene expression analysis revealed that CTBp had more pronounced impacts on the colon than the small intestine, with significant activation of TGF $\beta$-mediated pathways in the colon epithelium. The clinical relevance of CTBp-induced impacts on colonic mucosa was examined. In a human colon epithelial model using Caco2 cells, CTBp, but not the non-GM1-binding mutant G33D-CTBp, induced TGF $\beta$-mediated wound healing. In a dextran sodium sulfate (DSS) acute colitis mouse model, oral administration of CTBp protected against colon mucosal damage as manifested by mitigated body weight loss, decreased histopathological scores, and blunted escalation of inflammatory cytokine levels while inducing wound healing-related genes. Furthermore, biweekly oral administration of CTBp significantly reduced disease severity and tumorigenesis in the azoxymethane/DSS model of ulcerative colitis and colon cancer. Altogether, these results demonstrate CTBp's ability to enhance mucosal healing in the colon, highlighting its potential application in ulcerative colitis therapy besides cholera vaccination.

Cholera toxin B subunit (CTB) is the non-toxic, GM1ganglioside-binding subunit of cholera toxin $(\mathrm{CT}) .{ }^{1} \mathrm{CTB}$ is currently used in the World Health Organization-prequalified oral cholera vaccine Dukoral, due to its capacity to induce CTneutralizing antibodies. ${ }^{2}$ Because of its strong mucosal immunogenicity, CTB is often utilized as an adjuvant or a molecular scaffold in vaccine development. ${ }^{3}$ Additionally, previous studies suggested that CTB induces anti-inflammatory activities under immunopathological conditions. ${ }^{4}$ In a Phase I/II clinical trial, oral administration of CTB, chemically cross-linked to a peptide from the human $60 \mathrm{kDa}$ heat shock protein, alleviated uveitis of Behcet's disease. ${ }^{5}$ Orally administered CTB was also shown to blunt the intestinal inflammation of Crohn's disease. ${ }^{6,7}$ However, a comprehensive investigation of CTB's effect on the gastrointestinal (GI) tract has not been performed, thus leading to some debate on the protein's utilization in controlling the diseases of interest. The objective of this study is to characterize the impacts of orally administered CTB on the GI tract in detail, thereby attempting to fill the gap in our knowledge and facilitate the clinical applications of one of the most potent mucosal immunomodulators known to date.

To this end, we utilized a variant of CTB produced in Nicotiana benthamiana plants (CTBp), because the protein can be efficiently manufactured at scale, while showing GM1-binding affinity, physicochemical stability and oral immunogenicity for anti-toxin antibody induction comparable to original CTB. ${ }^{8,9}$ Thus, CTBp provides a viable alternative to the vaccine antigen included in Dukoral and potentially facilitates other clinical applications as described above. We first characterized the global impacts of CTBp oral administration on the small intestine and colon by elucidating

${ }^{1}$ Department of Pharmacology and Toxicology, University of Louisville School of Medicine, Louisville, Kentucky, USA. ${ }^{2}$ Owensboro Cancer Research Program of James Graham Brown Cancer Center at University of Louisville School of Medicine, Owensboro, Kentucky, USA. ${ }^{3} J a m e s$ Graham Brown Cancer Center, Department of Microbiology and Immunology, University of Louisville, Louisville, Kentucky, USA and ${ }^{4}$ Department of Medicine, University of Louisville School of Medicine, Louisville, Kentucky, USA. Correspondence: N Matoba (n.matoba@louisville.edu).

${ }^{5}$ These authors contributed equally to this work.

Received 25 January 2016; accepted 19 September 2016; published online 2 November 2016. doi:10.1038/mi.2016.95 
changes in their immune cell populations and gene expression profiles. The results led us to hypothesize that CTBp might induce TGF $\beta$-mediated mucosal wound healing, which was subsequently demonstrated in an in vitro human colon epithelial model using the Caco2 cell line. To examine the clinical relevance of these findings, we employed a dextran sulfate sodium (DSS) mouse model of intestinal wounding and ulcerative colitis, another major form of inflammatory bowel disease (IBD) along with Crohn's disease, to which CTB's influences have not been reported before. Furthermore, as mucosal healing potentially lowers colorectal cancer risk in ulcerative colitis, ${ }^{10}$ we investigated the effect of CTBp treatment on ulcerative colitis-associated tumorigenesis in the azoxymethane (AOM)/DSS model. Our data point to the potential utility of CTBp as an oral therapy for ulcerative colitis in addition to mass vaccination against cholera.

\section{RESULTS}

\section{Colon lamina propria leukocyte profile is significantly altered by CTBp oral administration}

Using flow cytometry, we characterized the immune cell populations of the lamina propria of small intestine and colon, Peyer's patches and spleen in mice two weeks after CTBp oral administration. The analysis revealed that subsets of innate immune cell populations in the lamina propria of colon (Figure 1) significantly increased; both $\mathrm{M} 1\left(\mathrm{CD} 80^{+}\right)$and M2 $\left(\mathrm{CD} 206^{+}\right)$subpopulations of macrophages $\left(\mathrm{F} 4 / 80^{+}\right)$, as well as natural killer $(\mathrm{NK})$ cells $\left(\mathrm{CD}_{4} 9 \mathrm{~b}^{+}\right)$were statistically significantly increased when compared with the control PBS group (Figure 1a and $\mathbf{b}$ ). The increase of these cell types was associated with a relative decrease in $B$ cells within the $\mathrm{CD} 5^{+}$cell population. Additionally, Th2, CD $25^{+} \mathrm{FoxP}^{+}$ T-regulatory (Treg) and Th17 cells were increased within the $\mathrm{CD}^{+}{ }^{+}$cell population in the colon of CTBp-administered group (Figure 1c). Increased macrophage infiltration into colon lamina propria on CTBp administration was confirmed by immunohistochemistry analysis (Figure 1d). Despite this, there was no abnormality or inflammation noted in the colon mucosa (data not shown). Meanwhile, such a major shift in immune cell profiles was not observed in the small intestine lamina propria, Peyer's patches or spleen (Supplementary Figure S1), indicating compartmentalized impacts of orally administered CTBp on immune cells in different regions of the GI tract.

The change in the colon immune cell profile made us speculate a possible change in the gut microbiota. However, the overall fecal microbiome profile showed no discernible shift at the point when the immune cell profiles were analyzed (Supplementary Figure S2). Bacteroidetes and Firmicutes spp. dominated at the phylum level, which is typical for C57BL/6J mice. ${ }^{11,12}$ There were significant changes at the species level in 12 Operational Taxonomic Units of minor subpopulations, with 11 belonging to the Clostridiales order (Supplementary Figure S3).
CTBp oral administration has a more pronounced effect on colon gene expression than small intestine

To further evaluate the impacts of orally administered CTBp on the GI tract, we performed microarray analysis of transcripts isolated from the small intestine and colon. The protein had profound impacts on the gene expression profile of both upper and lower GI tract (Figure 2 and Supplementary Figure S4). However, while gene expression profiles in small intestine samples clustered relatively tightly, colons from CTBp-treated mice showed a completely separated pattern compared with the control samples (Figure 2a). At a global level, 871 genes were significantly altered in the colon between CTBp and PBS groups $(P<0.01$; one-way ANOVA), while $\sim 5$ fold less (that is, 184$)$ genes were significantly altered in the small intestine (Figure 2b). Of these significantly altered genes, 539 were induced and 332 were suppressed in the colon. By comparison, the small intestine was fairly evenly split between induced and suppressed genes, with 97 and 87 altered genes, respectively, and there was no overlap with genes affected in the colon.

\section{CTBp enhances TGF $\beta$-associated gene expression pathways in the colon}

A gene expression pathway analysis (MetaCore version 6.22 build 67265) revealed that extracellular matrix (ECM) remodeling and epithelial to mesenchymal transition (EMT) pathways were among the most significantly induced pathways in the colon upon CTBp oral administration. In particular, TGF $\beta$-dependent pathways heavily populated the induced pathways (Figure 2c). Indeed, when evaluating individual gene expression from the microarray analysis $T g f b 1, T g f b I I$ receptor and Smad4 were found to be significantly induced by CTBp (Supplementary Figure S5), which is indicative of epithelial wound healing activity. ${ }^{13,14}$ By contrast, such strong induction of TGF $\beta$-related pathways was not observed in the small intestine. Suppressed pathways in the colon epithelium included several metabolic pathways, cystic fibrosis transmembrane conductance regulator (CFTR) pathways, and an apoptosis associated pathway. Genes associated with lipid, bile acid, pyruvate, and androstenedione and testosterone metabolic pathways were significantly blunted by CTBp. To confirm microarray data, quantitative real-time reverse-transcription PCR (qRT-PCR) was performed on selected induced, suppressed, or unaltered genes; a high agreement between microarray and qRT-PCR results was obtained (Supplementary Figure S6). Notably, several key genes in a wound healing pathway, including Colla1, Colla2, Col3a1, Col14a, Mmp2, Ctsk, Tagln, and Angpt1, were significantly upregulated by CTBp oral administration (Supplementary Figure S7).

\section{CTBp enhances wound healing in human colonic epithelial} cells

To investigate the mucosal wound healing potential of CTBp suggested by the gene expression analysis, we employed the human colon epithelial cell line Caco2 model. ${ }^{15}$ As shown in Figure $3 \mathbf{a}$ and $\mathbf{b}$, CTBp $(1.0$ and $3.0 \mu \mathrm{M})$ significantly enhanced wound closure, similarly to the TGF $\beta 1$ control. Indeed, 
increased levels of TGF $\beta 1$ and TGF $\beta 2$ were noted $24 \mathrm{~h}$ post wounding in the culture supernatant of CTBp treated cells (Supplementary Figure S8), and co-incubation of CTBp with an anti-TGF $\beta 1,2,3$ neutralizing antibody completely inhibited the wound healing activity (Figure 3c). Meanwhile, a nonGM1-binding CTBp variant, which has Gly33 $\rightarrow$ Asp mutation, ${ }^{16,17}$ failed to promote wound healing (Supplementary Figure S9), indicating that epithelial cell-surface GM1ganglioside is required for CTBp's wound healing activity. Analysis of the culture supernatant at $48 \mathrm{~h}$ revealed that CTBp and TGF $\beta 1$ had an overall similar inflammation and wound healing-related cytokine profile (Figure 3d); both produced similar levels of epidermal growth factor (EGF), fibroblast growth factor (FGF)-2, granulocyte-colony stimulating factor (G-CSF), granulocyte macrophage colony-stimulating factor (GM-CSF), interferon (IFN)- $\gamma$, interleukin (IL)-10, IL-1 $\beta$, IL-5, IL-6, tumor necrosis factor (TNF) $\alpha$ and vascular endothelial growth factor, in contrast to the PBS control. A major exception was monocyte chemoattractant protein-1, which was significantly elevated only in TGF $\beta 1$ treated cells.

\section{CTBp mitigates DSS-induced acute colonic injury and inflammation}

We next explored if the in vitro mucosal healing activity of CTBp could be translated into a therapeutic effect in vivo. We employed a well-established mouse DSS colitis model, which induces injury and severe inflammation in the distal colon. ${ }^{18} \mathrm{In}$ an initial study, PBS or $30 \mu \mathrm{g}$ CTBp was orally administered twice at a 2 week interval to mice before DSS exposure (Supplementary Figure S10). As shown in Figure 4a, CTBp significantly blunted the weight loss induced by DSS. Histopathological examination on hematoxylin and eosin (H\&E)-stained distal colon tissue at 6 days post DSS exposure revealed that CTBp treatment prevented the aberrant loss of crypts and ulceration that were noted in the untreated control group; although shortening of basal crypts and mild inflammatory infiltrates were observed, the epithelial surface remained intact (Figure 4b, c and Supplementary Figure S11). Despite the significantly less damage and inflammation in the colon of CTBp-administered mice, the numbers of macrophages infiltrated into the colon mucosa were similar between CTBp-treated and untreated groups, and significantly higher than that of the non-DSS-exposed control group (Figure 4d and $\mathbf{e}$ ), which might reflect the involvement of macrophages in the healing of the damaged tissue. ${ }^{19}$ Moreover, CTBp administration appeared to prevent fibrosis in the colon according to Masson's trichrome stain, while fibrosis was evident in the DSS-exposed vehicle control-administered group (Figure 4f). Consistent with this, the major fibrotic genes Colla1 and Tgfb1 (refs 20, 21) were significantly increased in the colon tissue of the DSS-exposed vehicle control-administered mice; CTBp treatment, on the other hand, showed lower levels of these transcripts (Figure 4g).

We also investigated CTBp's effect immediately after DSS exposure. At this maximum injury/inflammatory point, CTBp administration again significantly reduced the mucosal damage, characterized by shortened yet visible basal crypts, relatively mild inflammatory infiltrates in the mucosa and submucosa, and retention of the epithelial cell surface. Meanwhile, daily oral administration of $100 \mu \mathrm{g}$ mesalamine during the DSS exposure, which simulates a current treatment for ulcerative colitis in humans, ${ }^{22}$ showed similar protection observed in the CTBp-dosed group (Figure 5a and Supplementary Figure S12). In qRT-PCR analysis, CTBp treatment was shown to blunt the escalation of representative inflammatory marker expression, including Illb, Il33, Il6 and Infg (Figure 5b). Tgfbl, on the other hand, showed a significant increase in both CTBp-treated and vehicle control-administered mice following DSS exposure. Analysis of soluble markers in the distal colon also showed that CTBp administration blunted the significant increase of major inflammatory proteins, including IL-1 $\beta$, IL-3, IL-4, IL-5, IFN $\gamma$, IL-6, and GM-CSF (Figure 5c). Of note, CTBp administration did not increase IL-10 either at the gene expression or protein levels, despite that IL-10 has previously been linked to the potential anti-inflammatory activity of CTB. ${ }^{23,24}$

Since CTBp 'vaccination' was effective in DSS-induced acute colitis, we next examined the protein's therapeutic dosing effect. As shown in Figure 6, protection was evident as CTBp was dosed twice at the late phase of the DSS exposure (Day 3 and 6), when the onset of colonic epithelial damage had already taken place. ${ }^{25}$ This demonstrates that the protein does not require pre-emptive dosing for protection against DSS-induced colon epithelial insult. Conversely, CTBp's mucosal protective activity can take effect relatively quickly against ongoing epithelial damage and inflammation. A dose-ranging study (0.1-30 $\mu \mathrm{g}$ per dose $\times 2)$ showed that the most effective dose of CTBp in this therapeutic regimen was as low as two $1 \mu \mathrm{g}$ per dose. Interestingly, the efficacy did not follow a dose-dependent pattern; 3,10 , and $30 \mu \mathrm{g}$ were less effective than $1 \mu \mathrm{g}$, according to disease activity index (DAI) scores. Nevertheless, the highest dose still prevented the shortening of the colon length, showed a significantly lower inflammation score than the untreated control, and reduced epithelial damage and ulceration in the distal colon tissue, suggesting no adverse effects at this dose (Figure 6a-d). Consistent with the histopathological findings, analysis of distal colon tissue lysates showed that CTBp significantly suppressed pro-inflammatory cytokines/ chemokines (Figure 6e). Notably, a qRT-PCR array analysis revealed that CTBp significantly upregulated genes associated with wound healing (for example, epithelial repair, angiogenesis, microbial clearance), including Ctsg, (ref. 26) Angpt1, (ref. 27) Tagln, ${ }^{28} \operatorname{Itga1}$ (refs 27, 29) and Egf (refs 27, 29) (Figure 6f).

\section{CTBp oral administration can protect against chronic} colitis and colon tumor development in an AOM/DSS model The significant protection seen in the acute colitis/colon injury model prompted us to investigate if CTBp could also be effective in an AOM/DSS mouse model of chronic colitis and colon cancer. CTBp ( 3 or $10 \mu \mathrm{g})$ was given at the end of the first 
DSS exposure period followed by three additional doses every two weeks for a total of four doses (Supplementary Figure S13). As shown in Figure 7, CTBp administration $(3 \mu \mathrm{g})$ significantly decreased the DAI score immediately following the first dose and more dramatically during the third DSS exposure period (Figure 7a). Such a clear effect was not
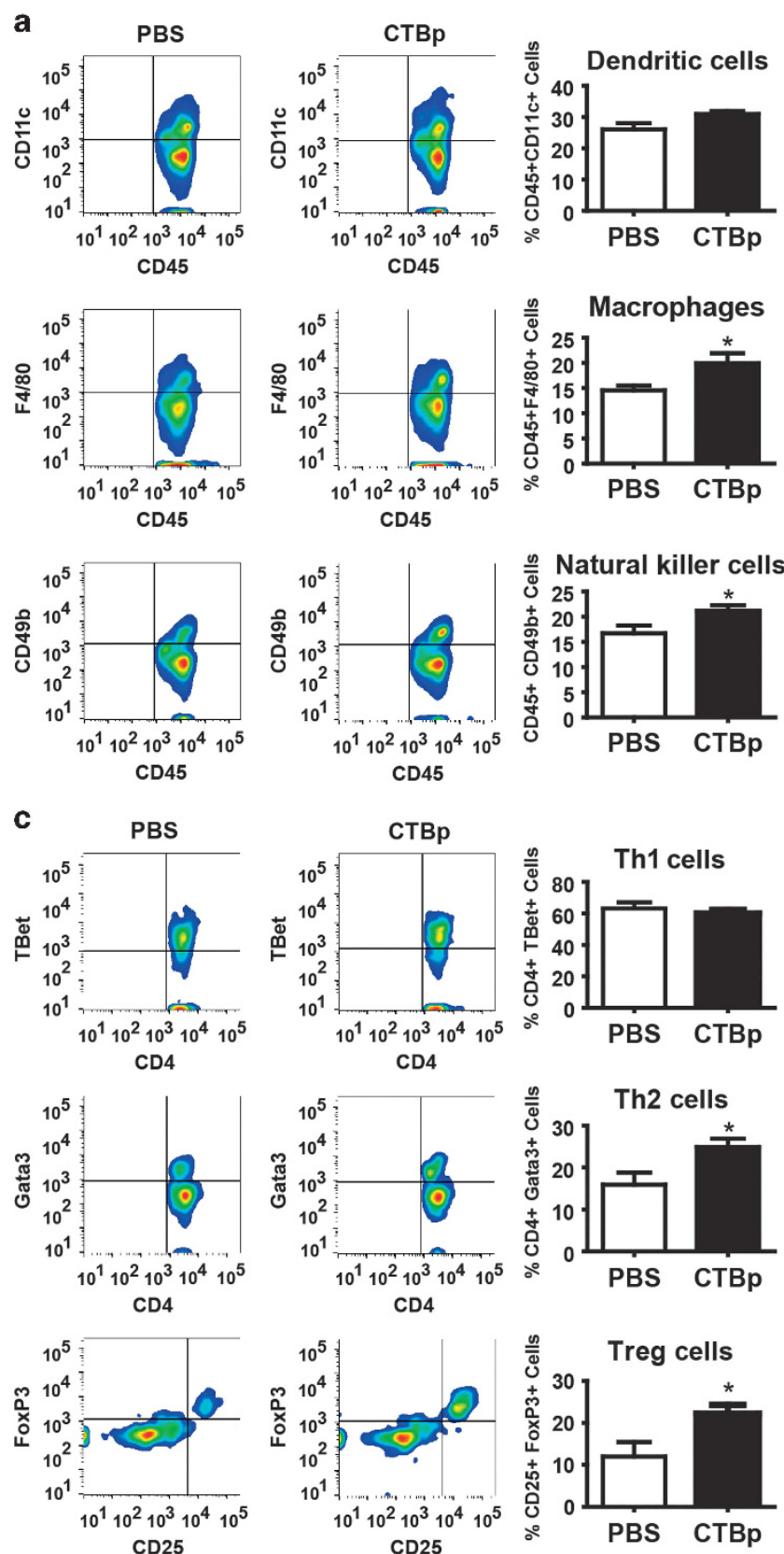
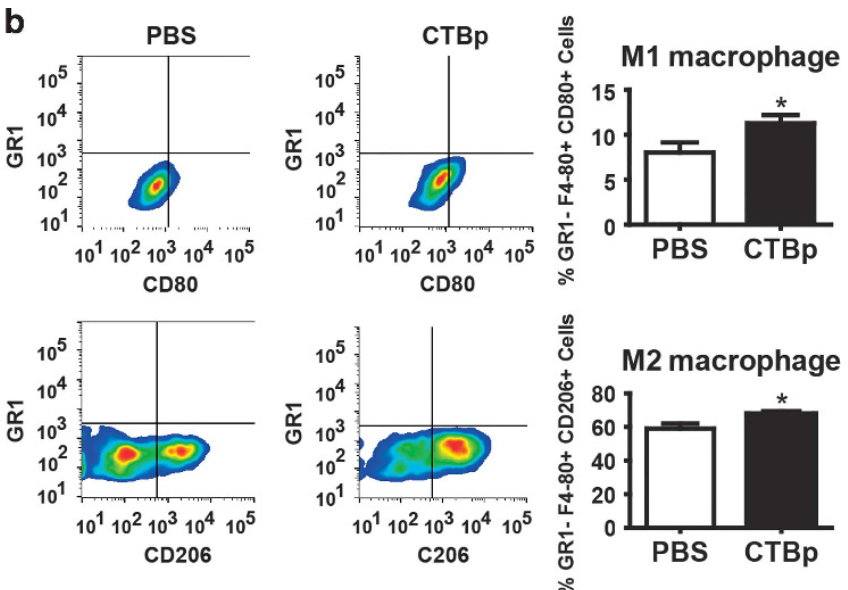

d

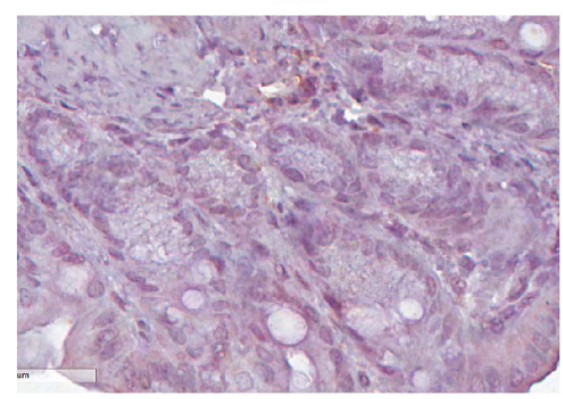

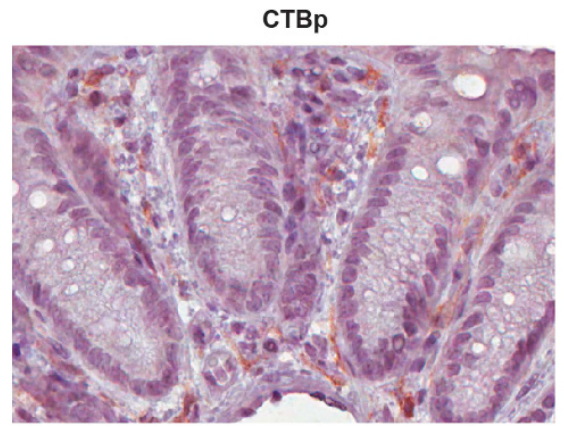
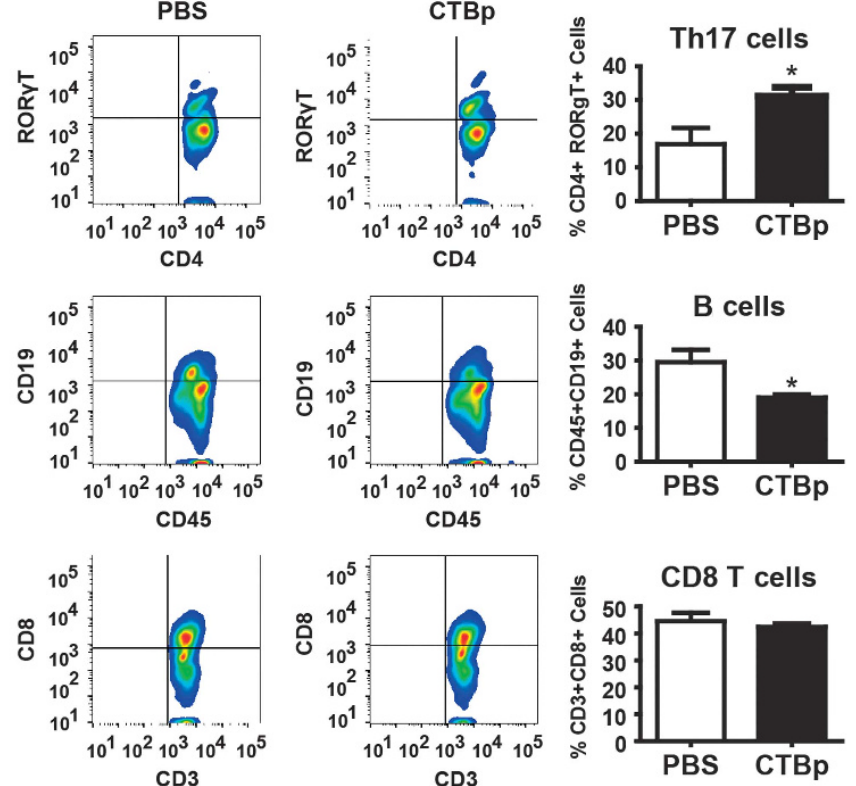
observed with the higher dose $(10 \mu \mathrm{g})$ of CTBp. However, tumorigenesis was significantly reduced at both dose levels (Figure 7c). Tumor growth also appeared to be limited by CTBp, as indicated by the decreased number of grade 3-5 tumors and total tumor grade in CTBp-administered groups (Figure $7 \mathrm{c}$ and d). Of note, CTBp administration $(10 \mu \mathrm{g})$ without DSS exposure did not induce any sign of intestinal damage or tumor growth (Figure $7 \mathbf{a}, \mathbf{c}, \mathbf{d}$ ).

To further characterize the protection of $3 \mu \mathrm{g}$ CTBp in the AOM/DSS model, we evaluated markers associated with inflammation and cancer development in the colon tissue two weeks following the final DSS exposure. Both DSS-exposed groups showed an increased level of Infg, Tgfb1, Foxp3, Il10, Nlrp3, Csf2, and Tnfa. However, CTBp administration resulted in a significant increase in Tgfb1, Foxp3 and Ifng compared with healthy mice (Figure 7e). Analysis of soluble markers showed that, although IL-1 $\beta$ had declined to a baseline level at this point, several inflammatory markers still showed a significantly higher level in the DSS-exposed, vehicle control-administered group compared with healthy mice, including $\mathrm{TNF} \alpha$, IL- $1 \alpha$,

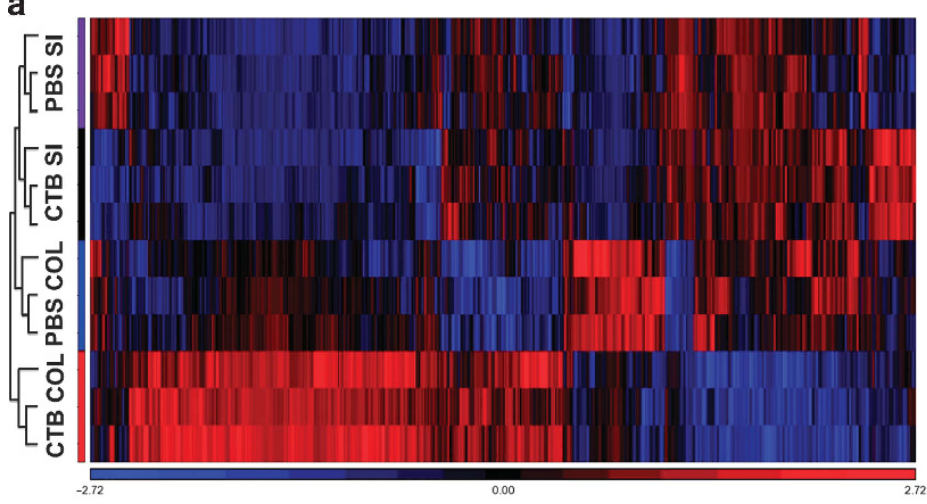

b

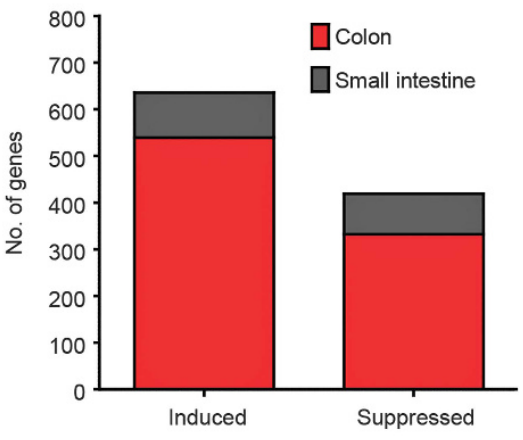

C

\begin{tabular}{|c|c|c|c|c|c|}
\hline Up Regulated Pathways & $p$ Value & Gene Ratio & Down Regulated Pathways & $\mathrm{p}$ Value & Gene Ratio \\
\hline $\begin{array}{l}\text { Cell Adhesion Extracellular Matrix Remodeling } \\
\text { (ECM) Remodeling }\end{array}$ & $5.97 \mathrm{E}-07$ & $10 / 52$ & $\begin{array}{l}\text { Androstenedione and Testosterone biosynthesis and } \\
\text { metabolism (Rodent version) }\end{array}$ & $6.838 \mathrm{e}-6$ & $7 / 36$ \\
\hline $\begin{array}{l}\text { TGF } \beta \text {-dependent induction of Epithelial to } \\
\text { Mesenchymal Transition (EMT) via SMADs }\end{array}$ & 2.07E-06 & $8 / 35$ & Oxidative phosphorylation & $9.240 \mathrm{e}-6$ & $11 / 105$ \\
\hline Regulation of EMT & 4.37E-06 & $10 / 64$ & $\begin{array}{l}\text { Cystic Fibrosis Transmembrane Conductance } \\
\text { Regulator (CFTR) folding and maturation }\end{array}$ & $5.341 \mathrm{e}-5$ & $5 / 21$ \\
\hline MicroRNA-dependent inhibition of EMT & 7.11E-06 & $5 / 12$ & $\begin{array}{l}\text { Androstenedione and Testosterone biosynthesis and } \\
\text { metabolism }\end{array}$ & $6.763 \mathrm{e}-5$ & $6 / 35$ \\
\hline $\begin{array}{l}\text { Normal and pathological TGF } \beta \text {-mediated regulation } \\
\text { of cell proliferation }\end{array}$ & 1.61E-04 & $6 / 33$ & $\begin{array}{l}\text { Regulation of Lipid metabolism PPAR regulation of } \\
\text { Lipid metabolism }\end{array}$ & $1.941 \mathrm{e}-4$ & $6 / 42$ \\
\hline TGF $\beta$-dependent induction of EMT via MAPK & $1.70 \mathrm{E}-04$ & $7 / 47$ & $\begin{array}{l}\text { Role of Diethylhexyl Phthalate and Tributyltin in fat } \\
\text { cell differentiation }\end{array}$ & $2.729 e-4$ & $5 / 29$ \\
\hline Cell adhesion Chemokines & 2.24E-04 & $10 / 100$ & Granzyme A signaling & $3.221 \mathrm{e}-4$ & $5 / 30$ \\
\hline
\end{tabular}

Figure 2 TGF $\beta$-dependent pathways are significantly altered by CTBp in the colon. PBS or CTBp was administered twice to mice (female C57BL/6J, 8-week old) at a two-week interval. Two weeks after the final dose animals were sacrificed and the small intestine and colon were removed for RNA purification. Total RNA was amplified and labeled, and then whole transcript expression analysis was performed. (a) Heat map showing differentially expressed genes in the small intestine (SI) and colon (COL) following PBS or CTBp administration. (b) Number of significantly altered genes in the colon and small intestine following PBS or CTBp administration. Significance was determined at $P<0.01$. (c) Ten most significantly enhanced and suppressed pathways by CTBp administration in the colon as determined by MetaCore ontologies enrichment analysis using $P<0.01$ and a fold-change of $<-1.2$ or $>1.2$.

Figure 1 CTBp significantly alters the immune cell profile in the colon. Animals (female C57BL/6J, 8-week old) were orally administered PBS or CTBp twice, at a two-week interval and two weeks later the mice were sacrificed. Colon lamina propria leukocytes were isolated and stained for surface and internal markers specific for immune cell subtypes. CD4 ${ }^{+}$and CD8 ${ }^{+}$cells gated on T lymphocyte subpopulation $\left(C D 45^{+}\right.$CD $\left.3^{+}\right)$. Additionally, CD45 ${ }^{+}$ cells were further divided into $B\left(\mathrm{CD}_{19}{ }^{+}\right)$, macrophage $\left(\mathrm{F} 4 / 80^{+}\right)$, dendritic $\left(\mathrm{CD} 11 \mathrm{c}^{+}\right)$and natural killer $\left(\mathrm{CD} 49 \mathrm{~b}{ }^{+}\right)$subpopulations. Dot plots are representative samples from each group. Data presented as mean \pm standard error of the mean (s.e.m.) of at least four biological replicates comprised of two pooled mice each. Unpaired $t$ test was performed with ${ }^{*} P<0.05$ compared with PBS group. (a) Innate immune cell populations in the colon lamina propria. (b) M1 (CD80 + ) and M2 (CD206 +) macrophage (F4/80 $\left.{ }^{+}\right)$populations in the colon lamina propria. (c) Adaptive immune cell populations in the colon lamina propria. (d) Immunohistochemistry analysis of macrophage $\left(\mathrm{F} 4 / 80^{+}\right)$cells in the distal colon lamina propria isolated from mice 2 weeks post second CTBp oral administration. Paraffin embedded colon sections were incubated with F4/80 primary antibody (1:100 dilution) and a biotinylated secondary antibody. After addition of a horseradish peroxidase (HRP) and 3,3'-diaminobenzidine tetrahydrochloride (DAB) solution, positive cells were counted in 10 high power fields per section and averaged for each colon. Mean \pm s.e.m. is shown. Unpaired $t$ test was performed with * $P<0.05$ compared with PBS group. Animals per group: PBS $(n=6)$ and $30 \mu \mathrm{g}$ CTBp $(n=7)$. 
a

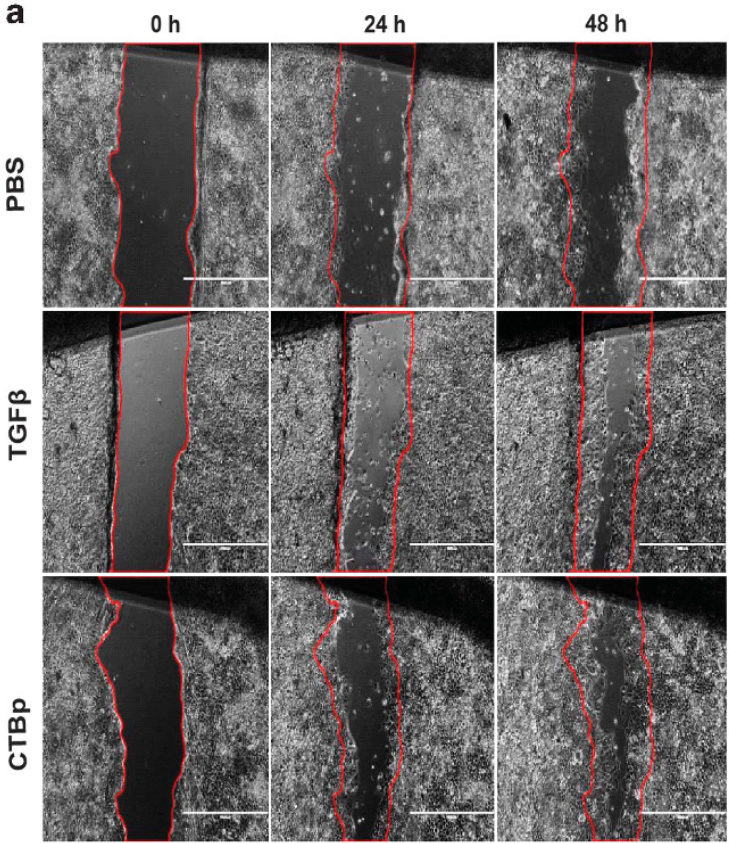

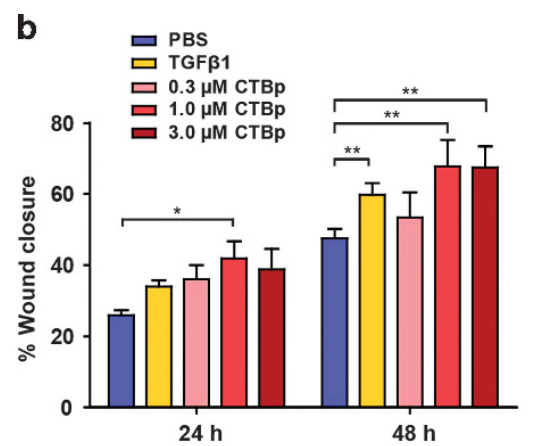

C

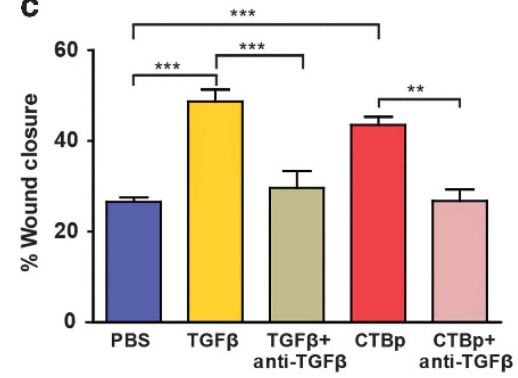

d
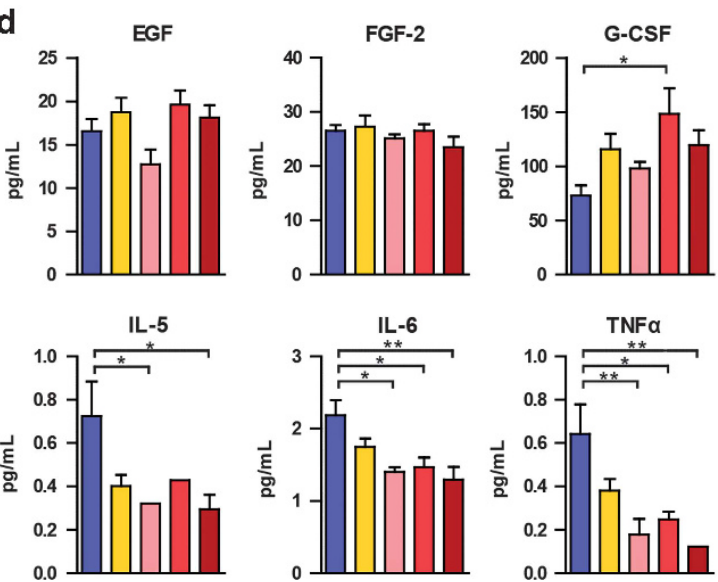
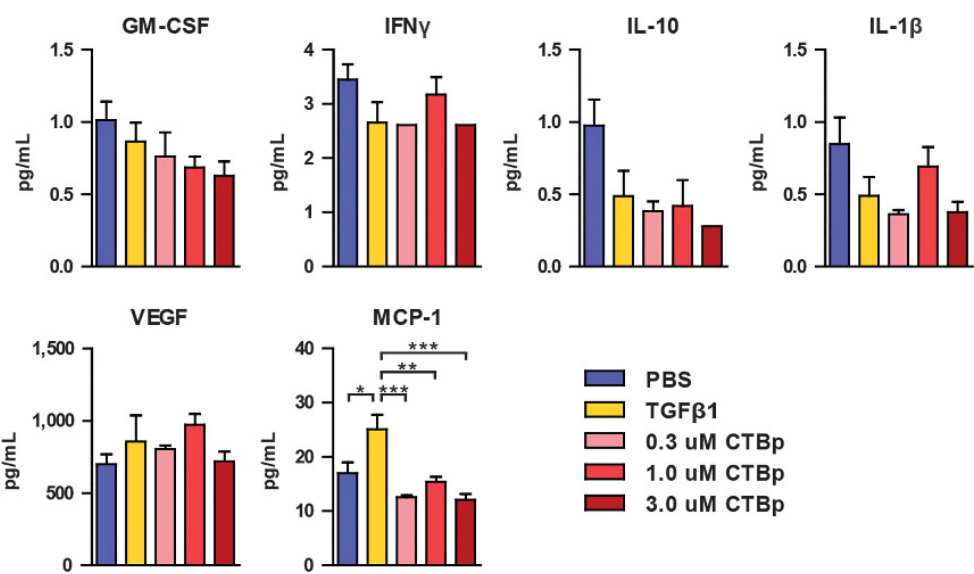

Figure 3 CTBp enhances wound healing in a human colon epithelial model. Caco2 cells were grown to confluence and scratched with a pipette tip. Cells were then incubated with PBS, TGF $\beta 1$, anti-TGF $\beta 1,2,3$ antibody, and/or CTBp. The in vitro wound closure was recorded over $48 \mathrm{~h}$ and $\times 4$ magnification images were acquired with a EVOS $f$ l by Advanced Microscopy Group and mean percentage closure was determined by Image $\mathrm{J}$ software.

(a) Photomicrographs of wounded Caco2 cells. (b) Analysis of in vitro wound closure after 24 and $48 \mathrm{~h}$ by wound area measurement. (c) Analysis of in vitro wound closure after $24 \mathrm{~h}$ by wound area measurement after incubation with PBS or an anti-TGF $\beta 1,2,3$ antibody coincubated with $1.0 \mu \mathrm{M}$ CTBp or $0.2 \mathrm{nM}$ TGF $\beta 1$. (d) Protein concentrations in Caco2 cell supernatants. Means \pm s.e.m. of four independent experiments are shown (b, c, d).

One-way ANOVA with Bonferroni's multiple comparison tests was used to compare all pairs of groups (b, $\mathbf{c}, \mathbf{d})$. Significantly different pairs are highlighted with asterisks $\left({ }^{*} P<0.05,{ }^{* \star} P<0.01\right.$ and $\left.{ }^{* * *} P<0.001\right)$.

keratinocyte chemoattractant, chemokine (C-X-C motif) ligand 9 (Figure 7f). CTBp administration blunted the elevation of these markers, most notably IL- $1 \alpha$, which is a key factor to exacerbate gut inflammation. ${ }^{30}$ Notably, CTBptreatment completely blocked the AOM/DSS-induced decrease of IFN $\gamma$ and IL-2 levels, which could be linked to the reduced tumorigenesis in this group. ${ }^{31,32}$

\section{DISCUSSION}

Oral administration of CTB leads to a robust antibody response and an anti-inflammatory effect. ${ }^{3,4}$ The former represents the protein's most well-known biological activity, which has been exploited in cholera prevention. ${ }^{2}$ On the other hand, the utilization of CTB's anti-inflammatory activity in inflammatory disease therapy is yet to be achieved, in part due to its obscure underlying mechanisms. The present study revealed that oral administration of CTBp exhibits previously unidentified impacts on the distal part of the GI tract; modification of the immune cell profile and upregulation of TGF $\beta$ pathways in the colon (Figure 1 and 2), which subsequently lead us to discover that CTBp promotes mucosal healing in the colon (Figures 3-7). Although CTB was previously shown to upregulate TGF $\beta$ in immune cells $s^{4,33}$ and blunt intestinal inflammation of Crohn's disease, ${ }^{6,7}$ the 


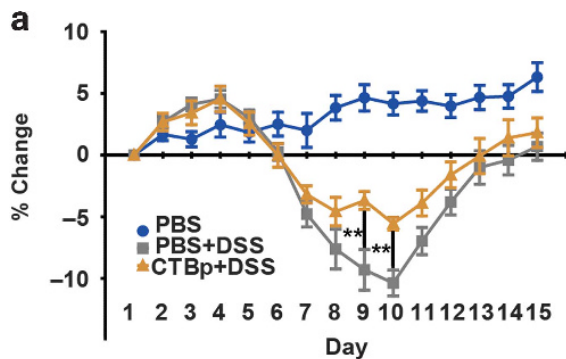

b
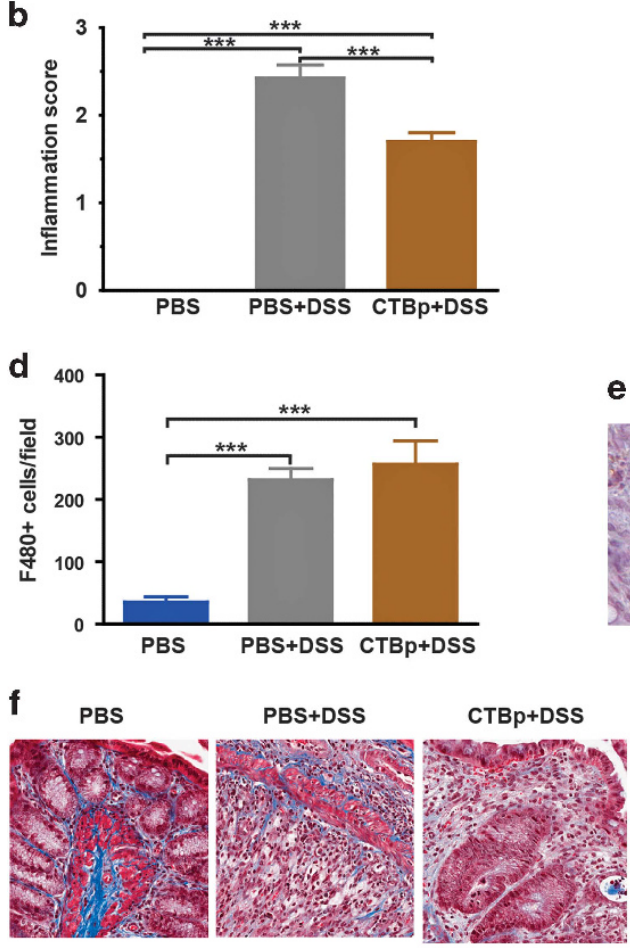

e
C
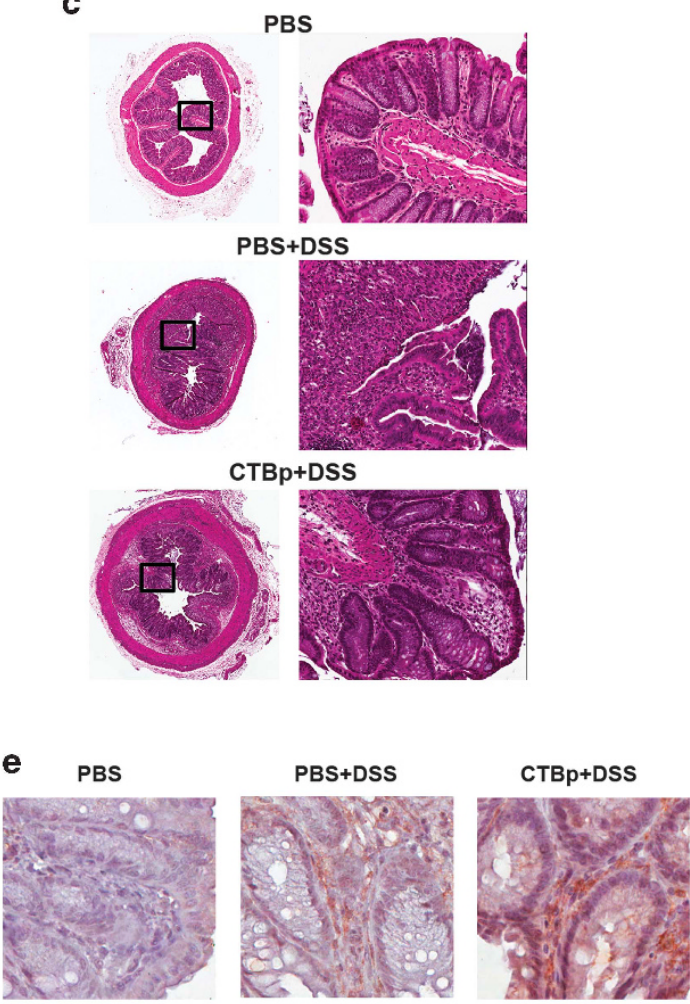

g

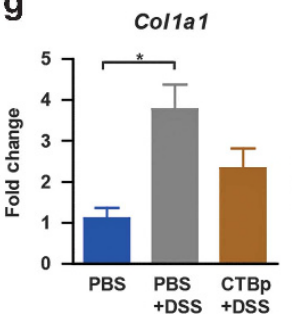

Figure 4 Oral administration of CTBp blunts DSS-induced colonic epithelial damage. Mice (female C57BL/6J, 8-week old) were orally administered PBS or CTBp twice at a two-week interval. Immediately after the second administration DSS exposure began and continued for 8 days. Colon tissues were isolated after a 6-day recovery for analyses. Mean \pm s.e.m. is shown for each group. Animals per group: PBS $(n=6), 30 \mu \mathrm{g}$ CTBp $+\mathrm{DSS}=(n=8)$, and PBS + DSS $(n=8)$. (a) Percent change of body weights. Animals were weighed daily and just prior to the initiation of DSS exposure. Percent change was based on the initial body weight. ${ }^{* *} P<0.01$ between DSS-exposed, CTBp and PBS-administered groups; two-way ANOVA with Bonferroni's multiple comparison tests. (b) Colon inflammation scoring. Paraffin embedded tissue sections were scored after staining with Hematoxylin and Eosin (H\&E). Scoring was based on 0-4 scale. (c) Representative $\times 4$ (left) and $\times 20$ (right) photomicrographs of colons from treatment groups. (d, e) Immunohistochemistry staining of $\mathrm{F} 4 / 80^{+}$cells in distal colon tissue. Means \pm s.e.m. of positive cells from 10 individual microscope fields per sample (d) and representative photographs (e) are shown. (f) Representative photomicrographs of colons following Masson's Trichrome Stain. (g) qRT-PCR analysis of cytokine gene expression in mouse colon tissue. Mean \pm s.e.m. is shown for each group $(N=5)$. For $(\mathbf{b}$, $\mathbf{d}$, g), one-way ANOVA with Bonferroni's multiple comparison tests was used to compare all pairs of groups. Significantly different pairs are highlighted with asterisks $\left({ }^{*} P<0.05\right.$, ${ }^{* *} P<0.01$, and $\left.{ }^{* * *} P<0.001\right)$.

present studies demonstrated for the first time that orally administered CTB can facilitate mucosal wound healing in the colon.

In the mouse acute colitis model, CTBp oral administration significantly reduced ulceration in colon epithelia (Figures 4-6), which is indicative of enhanced mucosal healing. ${ }^{10,34}$ The escalation of inflammatory markers was blunted, while $T g f b 1$ expression significantly increased (Figures $\mathbf{5 b}$ and $\mathbf{c}$ ). Given that CTBp-induced TGF $\beta$-mediated wound healing in Caco2 colon epithelial cells via GM1-ganglioside binding (Figure 3 and Supplementary Figure S9) and previous studies demon- strated that epithelial cell-derived TGF $\beta$ promotes colon mucosal healing, ${ }^{14,35}$ it is strongly suggested that CTBp's mucosal healing effect in the DSS model is primarily ascribed to epithelia-derived TGF $\beta$. A recent study showed that CFTR regulation of GM1 is involved in airway epithelial wound healing. ${ }^{36}$ Meanwhile, we showed that oral administration of CTBp upregulated a pathway related to sphingolipid transport and recycling, while down-regulating CFTR-related pathways in the colon (Figure 2c). Thus, our findings point to a potential mechanistic link between CFTR, GM1, and TGF $\beta$ signaling in CTBp-induced colon mucosal healing. 
TGF $\beta$ has multifaceted functions including pivotal roles in gut homeostasis and intestinal wound healing. ${ }^{29,37} \mathrm{~A}$ recent study by Oshima et al. ${ }^{38}$ has shown that the suppression of
TGF $\beta$ signaling in an injured and inflamed mucosa leads to invasive tumor development in the colon, showing that TGF $\beta$ mediated mucosal repair plays a key role in colitis-associated a

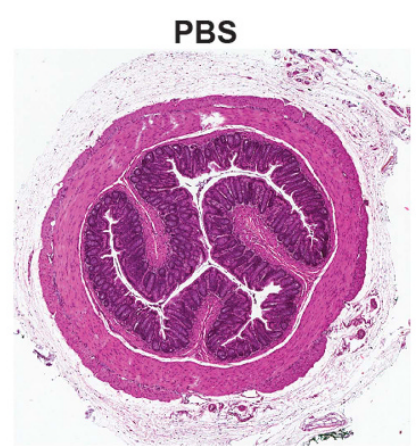

MES + DSS

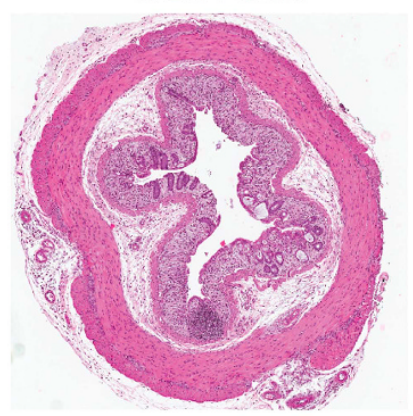

CTBp

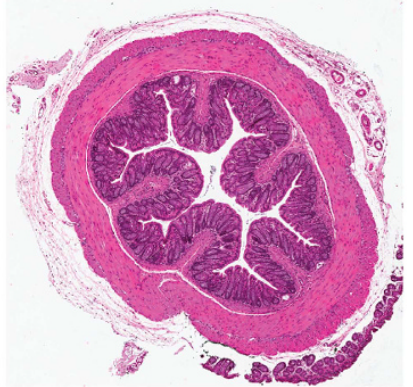

CTBp + DSS
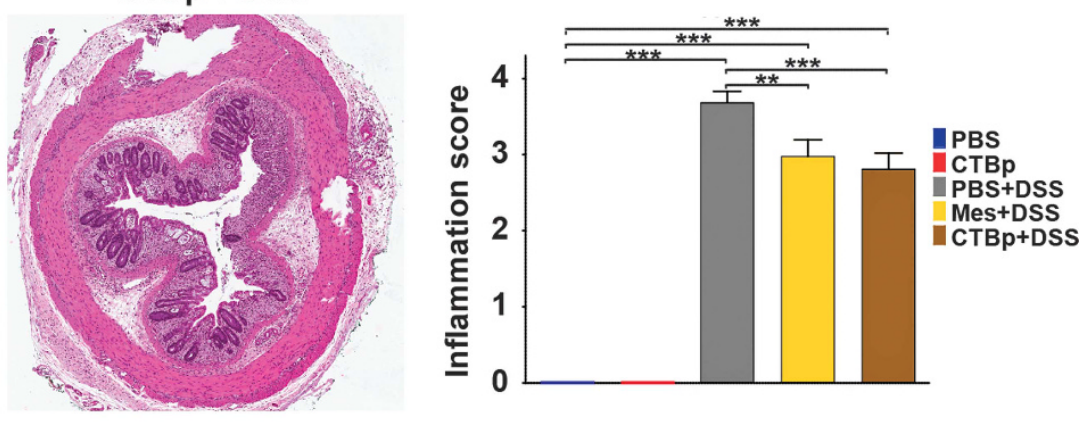

b
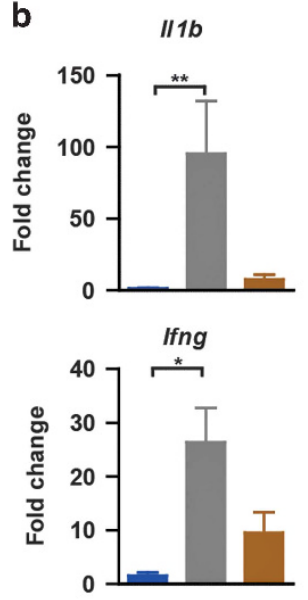

c

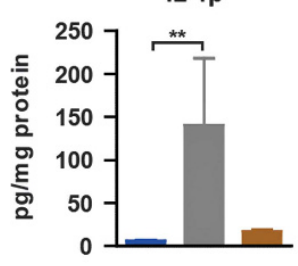

IFNy

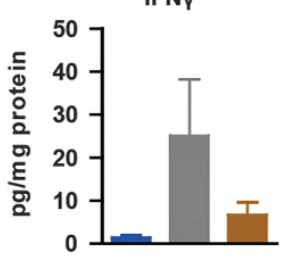

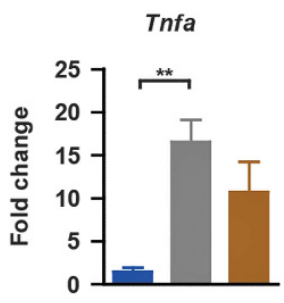

$1 / 6$

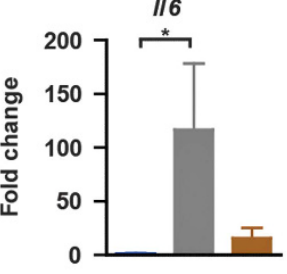

TNFa
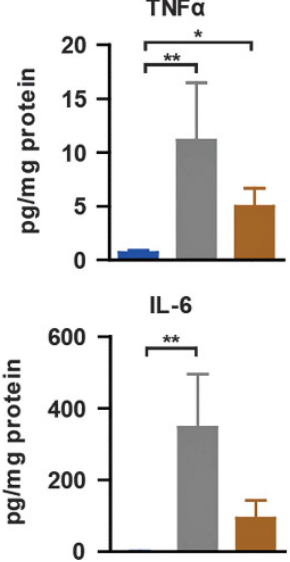
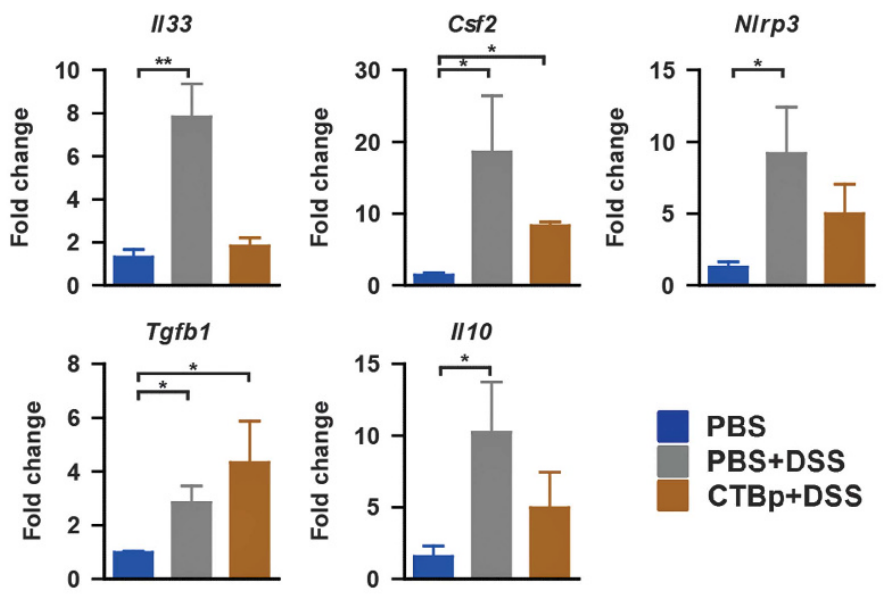

IL-3
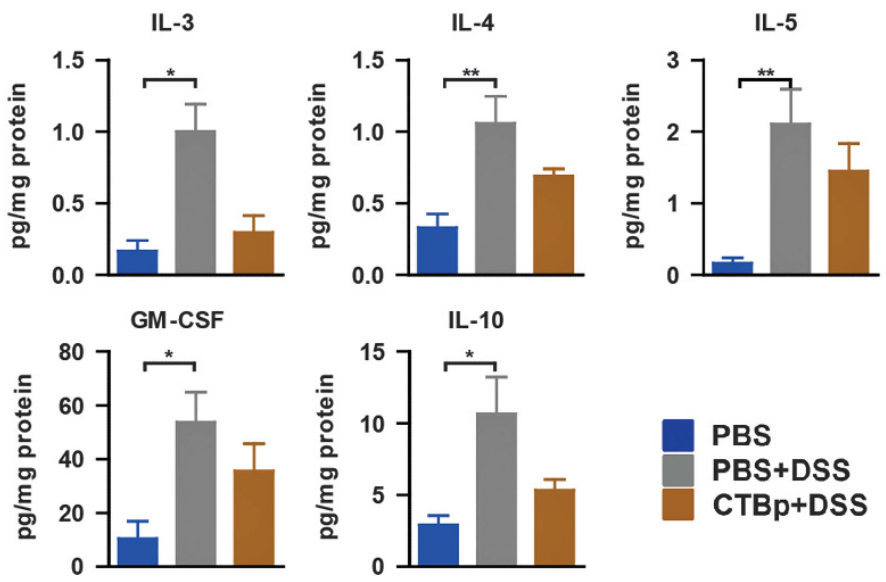

PBS

PBS+DSS
CTBp+DSS 
colon tumor prevention. This is in line with our findings in the AOM/DSS study (Figure 7). However, TGF $\beta 1$ is also known to be elevated in collagenous colitis and connected to increased collagen deposition and fibrosis, indicating the dual nature of this cytokine in mucosal remodeling. ${ }^{37}$ In this regard, the fact that CTBp administration prevented fibrosis in the acute DSS colitis study (Figures $\mathbf{4} \mathbf{f}$ and $\mathbf{g}$ ) suggests that our CTBp dosing regimen did not overstimulate TGF $\beta$ signaling to a level causing adverse effects. Indeed, $T g f b 1$ was high in the early inflammatory phase (Figure $\mathbf{5 b}$ ) and became lower in the recovery phase (Figure $\mathbf{4 g}$ ) in the CTBp-treatment group. The apparent lack of dose dependence in CTBp-induced TGF $\beta$ levels and wound healing effects might be indicative of hormesis, a biphasic dose response characterized by lowdose stimulation and high-dose inhibition. ${ }^{39}$ Such responses were observed with wound healing agents/stimulations, ${ }^{40}$ as well as recombinant IL-10 and anti-TNF $\alpha$ monoclonal antibody in Crohn's disease clinical studies. ${ }^{41,42}$ This may explain why the higher doses of CTBp were not as effective as the lower dose in the acute and chronic DSS studies (Figures 6 and 7). Detailed investigation into CTBp's dose-effect relationship is warranted to define a therapeutic window for optimal mucosal healing.

It is of interest to note that CTBp-induced TGF $\beta$ activation in the colon epithelium was accompanied by a significant increase of M1/M2 macrohpages and NK cells in the same mucosa (Figures 1 and 2). CTB is known to alter the T cell profile under various conditions and affect DC maturation in vitro. ${ }^{4}$ However, the protein's impacts on immune cells in different regions of the GI tract, as shown in the present study, are unprecedented. At this point, the mechanism by which CTBp-induced such compartmentalized effects on GI tract immune cells is not clear, and the gut microbiota was unlikely the cause of the drastic changes in the colon (Supplementary Figure S2 and S3). One possibility is that CTBp's effects might have remained longer in the colon, as epithelial turnover is slower in the colon than the small intestine. ${ }^{43}$ Meanwhile, the observation that CTBp concomitantly stimulated the activation of TGF $\beta$ signaling and the increase of the innate immune cells in the colon leads us to postulate that the latter effect might also play a role in the protein's mucosal healing effects. In fact, mucosal healing is a dynamic biological process involving a number of mediators and cell types, including immune cells. ${ }^{27,29}$ For example, NK cells play a major role in tissue remodeling by clearing dead or dying cells. ${ }^{44}$ Macrophages can remove bacteria that penetrate the epithelium and damaged tissue and play an important role in enhancing wound healing. ${ }^{19}$ Studies are currently underway to analyze the pharmacokinetics and biodistribution of orally administered CTBp in the GI tract and the potential interplay of epithelial and immune cells in CTBp-induced colon mucosal healing.

Since DSS colitis is pathologically similar to ulcerative colitis in humans, ${ }^{45}$ our data point to a possibility that CTBp is effective against ulcerative colitis. In particular, mucosal healing has recently become an important target for ulcerative colitis therapy because it is associated with improved clinical outcomes. ${ }^{10,29}$ It should be noted that only two low oral doses of CTBp were as effective as mesalamine dosed daily during the 8-day DSS exposure in the acute colitis model (Figure 5), and biweekly dosing of CTBp also proved to be effective against chronic colitis and reduced colitis-associated colon tumorigenesis (Figure 7), highlighting the protein's remarkable therapeutic potential.

In summary, our work revealed a novel function of CTBp to enhance colonic mucosal healing through TGF $\beta$ pathways. Although further investigations are required to carefully define the dose-effect relationship in mucosal remodeling and efficacy in other clinically relevant conditions such as Smad7 overexpression, ${ }^{29}$ an efficient bioproduction system already available for CTBp (ref. 8) should significantly facilitate the protein's preclinical and clinical investigations towards its potential use to promote colonic mucosal health besides cholera prevention.

\section{METHODS}

Animals. Eight-week-old C57BL/6J female mice were obtained from Jackson Laboratories (Bar Harbor, ME). Animal studies were approved by the University of Louisville's Institutional Animal Care and Use Committee.

Study design. For all animal experiments, five to nine mice per group, randomly assigned, were used. For the characterization of the global impacts of CTBp oral administration, animals were gavaged with PBS or $30 \mu \mathrm{g}$ CTBp twice at a 2-week interval after sodium bicarbonate administration, as described previously. ${ }^{8}$ CTBp was produced in $N$. benthamiana and purified to $>95 \%$ homogeneity with an endotoxin level of $<1$ endotoxin units per $\mathrm{mg}$, as described previously. ${ }^{8}$ Two weeks after the second dose, mice were sacrificed, and feces, colon, small intestine, spleen and Peyer's patches were collected. For the acute DSS 'vaccination' study, DSS exposure was initiated on the day of the second dosing (Supplementary Figure S10), using a modified method. ${ }^{46}$ Body weights were measured at the initiation of DSS exposure as a baseline and every morning thereafter to determine percent change. Animal DAI scores were determined daily as previously described. ${ }^{46}$ Animals received 4\% DSS (M.W. 36,000 to 50,000;

Figure 5 CTBp significantly reduces colon inflammation induced by DSS exposure. Mice (female C57BL/6 J, 8-week old) were orally administered with PBS or CTBp and exposed to DSS as in Figure 4. As a reference control, a group of mice were treated with oral administration of $100 \mu \mathrm{g}$ mesalamine daily during the DSS exposure. Colon tissues were isolated immediately after the DSS exposure for analyses. (a) Representative photomicrographs of colons from the treatment groups and colon inflammation scoring. Paraffin embedded tissue sections were scored after staining with H\&E. Scoring was based on 0-4 scale. Mean \pm s.e.m. is shown for each group. Animals per group: PBS $(n=8), 30 \mu \mathrm{g}$ CTBp + DSS $(n=8)$, mesalamine + DSS $(n=9)$ and PBS + DSS $(n=7)$. (b) qRT-PCR analysis of cytokine gene expression in mouse colon tissue. Mean \pm s.e.m. is shown for each group $(N=5)$. (c) Cytokine concentrations in colon tissue lysate. $N=5$ per group. One-way ANOVA with Bonferroni's multiple-comparison post-test (a) or Kruskal-Wallis test with Dunn's multiple-comparison post-test $(\mathbf{b}, \mathbf{c})$ were used to compare groups for all pairs. Significantly different pairs are highlighted with asterisks $\left({ }^{*} P<0.05,{ }^{* *} P<0.01\right.$, and $\left.{ }^{* * *} P<0.001\right)$. 


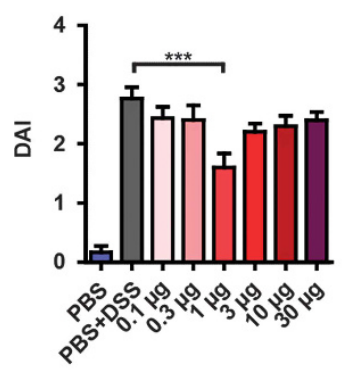

b

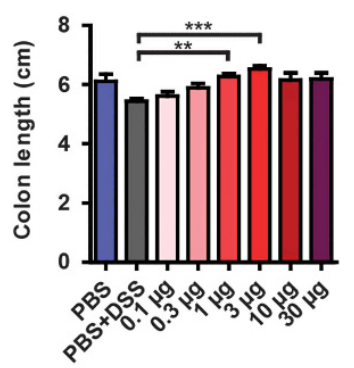

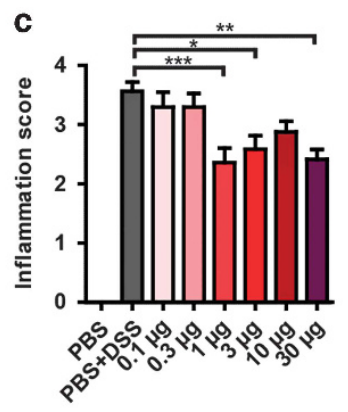

d
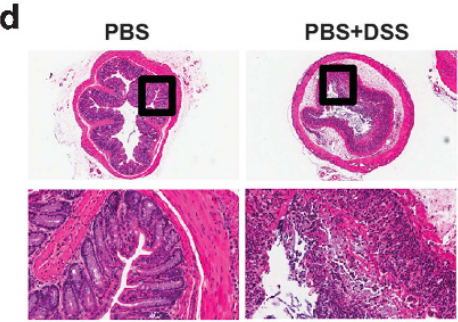

$0.1 \mu \mathrm{g}$ СТВp
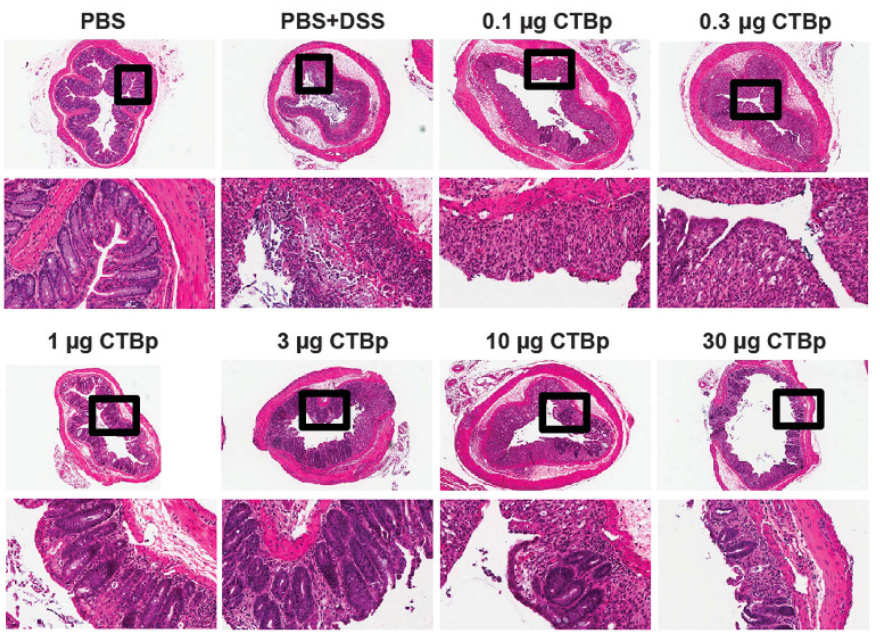

$3 \mu \mathrm{g}$ CTBp
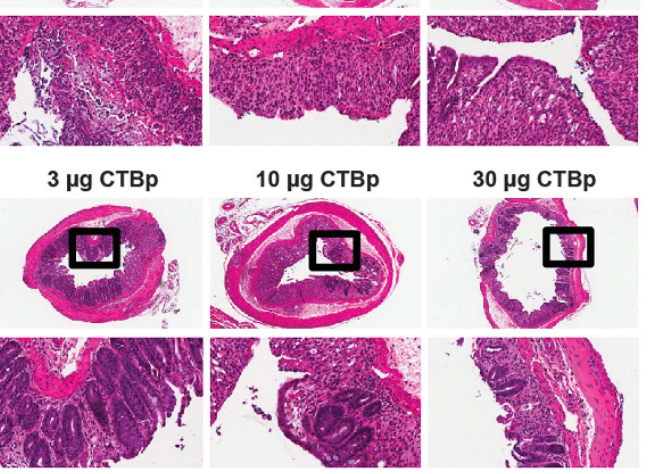

e
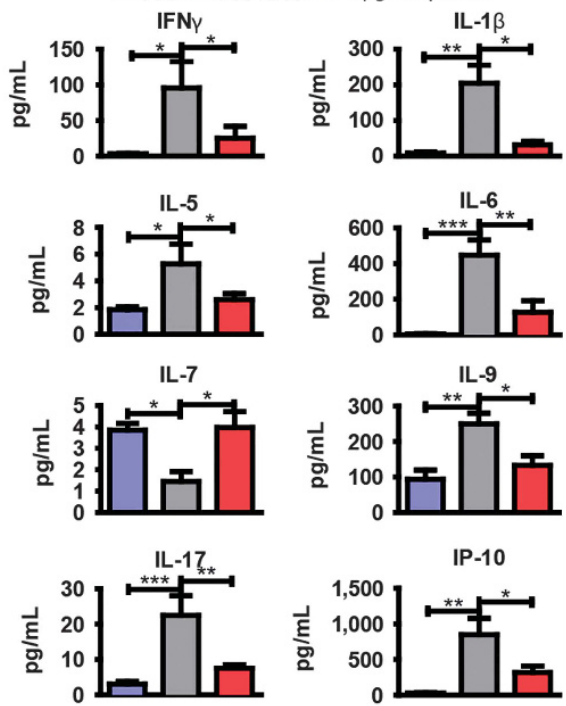

IP-10
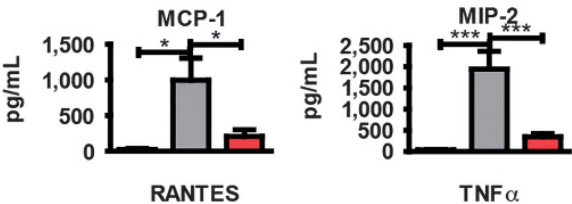

$\mathrm{TNF} \alpha$
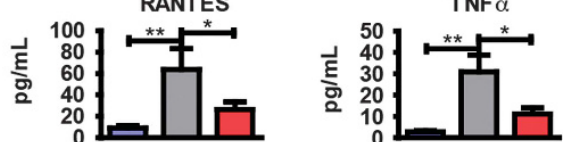

$\mathbf{f}$

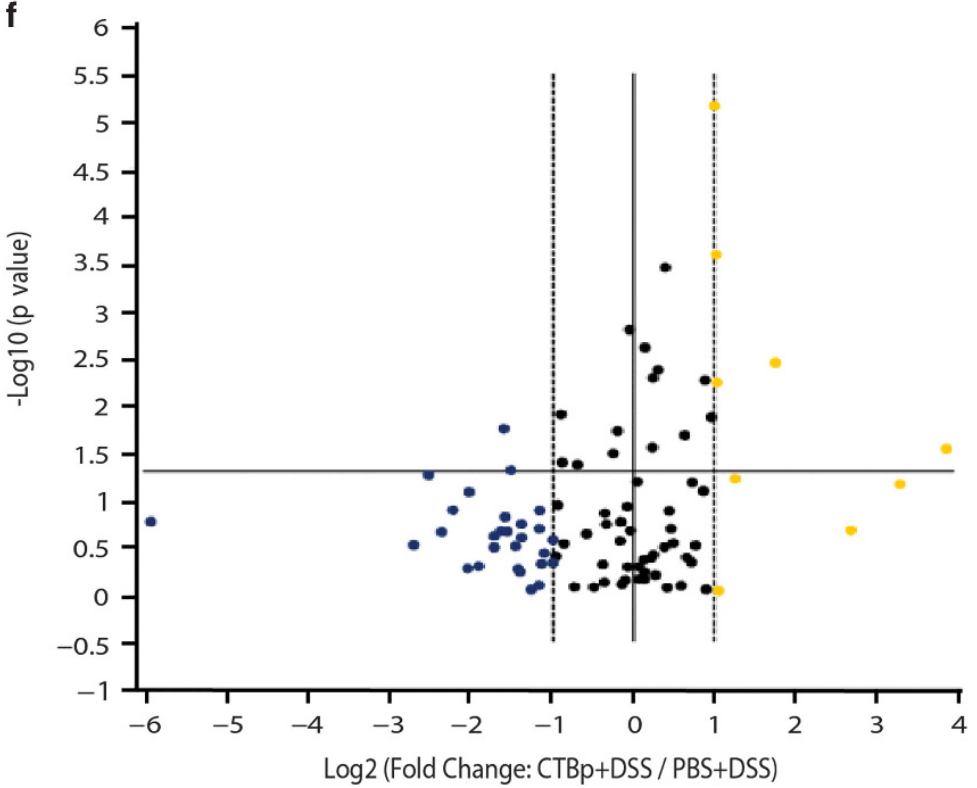

\begin{tabular}{|c|c|c|}
\hline Gene & p Value & $\begin{array}{c}\text { Fold } \\
\text { regulation }\end{array}$ \\
\hline Cstg & 0.029 & 14.5 \\
\hline Angpt1 & 0.004 & 3.37 \\
\hline Tagln & 0.006 & 2.04 \\
\hline Itga1 & 0.000 & 2.03 \\
\hline Egf & 0.000 & 2.00 \\
\hline Fgf10 & 0.068 & 9.75 \\
\hline
\end{tabular}

Upregulated Unchanged ODownregulated

Figure 6 Therapeutic dosing of CTBp is effective in ameliorating DSS-induced acute colitis. Mice (female C57BL/6 J, 8-week old) were exposed to $3 \%$ DSS for seven days and orally administered with PBS or CTBp on the third and sixth day. Colon tissues were isolated after a two-day recovery for analyses. $N=5$ for PBS and $N=10$ for all other groups. (a) Disease activity index (DAl) scores. Body weight loss, fecal consistency and occult blood were scored at the time of sacrifice. (b) Colon length. (c) Colon inflammation scoring. Paraffin embedded tissue sections were scored after staining with H\&E. Scoring was based on 0-4 scale. Mean \pm s.e.m. is shown for each group (d) Representative $\times 4$ (top) and $\times 20$ (bottom) photomicrographs of H\&Estained distal colon tissues from each group. (e) Cytokine concentrations in colon tissue lysate. $N=5$ per group. (f) qRT-PCR analysis of gene expression in mouse colon tissue. The graph shows $P$ value vs. fold-change. Dots represent 2 -fold upregulation (yellow), 2 -fold down-regulation (blue), or $<2$-foldchange (black). Values (right table) were calculated by the data analysis web portal at http://www.qiagen.com/geneglobe $(N=4)$. One-way ANOVA with Bonferroni's multiple comparison tests was used to compare all CTBp treatment groups to PBS + DSS control group for (a, b, c) and all pairs of groups for (e). Significantly different pairs are highlighted with asterisks $\left({ }^{*} P<0.05,{ }^{* *} P<0.01\right.$, and $\left.{ }^{* * *} P<0.001\right)$. 
MP Biomedicals, Santa Ana, CA) in drinking water for 8 days, and allowed to recover 6 days during which the animals received normal drinking water. For the therapeutic dose ranging study, mice were orally administered with PBS or CTBp twice (day 3 and 6) during DSS exposure. Animals were exposed to 3\% DSS for 7 days and allowed 2-day recovery. For the AOM/DSS study (Supplementary Figure S13), $\operatorname{AOM}\left(10 \mathrm{mg} \mathrm{kg}^{-1}\right)$ was administered by intraperitoneal injection. DSS exposure (2\%) was initiated 1 week after the AOM injection for 7 days and allowed to recover for 14 days. The DSS exposure and recovery cycle was repeated 3 times and mice were sacrificed following the third cycle.

Immune cell isolation. Lamina propria lymphocytes were isolated from the colons and small intestines as previously described. ${ }^{47,48}$ The intestinal tissues were incubated with EDTA at $37^{\circ} \mathrm{C}$ and cut into small pieces and incubated with collagenase at $37^{\circ} \mathrm{C}$. Splenocytes were isolated by crushing the spleens on metal mesh and separating the supernatant. Ammonium chloride potassium carbonate buffer was added and following several washes the cells were filtered through a $70 \mu \mathrm{m}$ cell strainer. Peyer's patch were minced and incubated in collagenase at $37^{\circ} \mathrm{C}$ twice. After a second wash the cells were combined and filtered through a $70 \mu \mathrm{m}$ cell strainer.

Flow cytometry. Immune cells from 2 mice were pooled for each biological replicate with a total of at least 4 biological replicates per group. Briefly, tubes containing $1 \times 10^{6}$ cells were washed with Flow Cytometry Staining Buffer (FCSB) 2 times. Fc Block was added to each tube in FCSB for $10 \mathrm{~min}$. For CD4 cell populations, cells were incubated with surface staining antibodies (anti-CD3-FITC, antiCD4-APC-Cy7, anti-CD25-PerCP) at $4{ }^{\circ} \mathrm{C}$ for $30 \mathrm{~min}$. After removing excess antibodies, Fixation/Permeabilization Buffer (eBiosciences, Inc.) was added and incubated overnight. Cells were washed and incubated with Fc block. Internal cell antibodies (Gata3-PE, T-Bet-PECy7, FoxP3-APC, IL-17-eFlour450) were then added and incubated for $30 \mathrm{~min}$ at $4{ }^{\circ} \mathrm{C}$. Finally, cells were washed and suspended in FCSB. For other immune cell populations, surface staining antibodies (CD19-APC, CD3-FITC, CD49b-PE, F4/80-PeCy7, CD11c-PerCPCy5.5, CD8-APC-eFluor 780, and CD45-eFlour450 or CD45-APCCy7, CD11b-PerCP-Cy5.5, CD11c-FITC, F4/80-APC, CD80-Pacific Blue or isotype control, and GR1-PE) and internal cell antibodies (CD206-PeCy7 or isotype control) were used. Events $\left(1 \times 10^{5}\right)$ were counted on a BD FACSCanto II and analyzed with the BD FACSDiva Software v6.1.3.

RNA isolation. Sections from the small intestine and distal colon were stored in RNAlater (Qiagen, Valencia, CA) at $-20{ }^{\circ} \mathrm{C}$ until RNA was isolated. Colon tissue ( $\sim 14 \mathrm{mg}$ ) was placed in QIAzol lysis reagent and homogenized. An RNeasy Microarray Tissue Kit from Qiagen was used to purify the RNA from the tissue homogenate. RNA was stored at $-80^{\circ} \mathrm{C}$ until use.

Microarray gene expression analysis. Total RNA was amplified and labeled following the Affymetrix (Santa Clara, CA) standard protocol for whole transcript expression analysis, followed by hybridization to Affymetrix Mouse Gene $2.0 \mathrm{ST}^{\circledR}$ arrays. The arrays were processed following the manufacturer recommended wash and stain protocol on an Affymetrix FS-450 fluidics station and scanned on an Affymetrix GeneChip 7G scanner using Command Console 3.3. The resulting.cel files were imported into Partek Genomics Suite 6.6 and transcripts were normalized at the gene level using RMA as normalization and background correction method. ${ }^{49}$ Contrasts in a one-way ANOVA were set up to compare the treatments of interest.

qRT-PCR. First strand cDNA was obtained from reverse transcription of $150 \mathrm{ng}$ RNA using a SUPERSCRIPT VILO cDNA synthesis kit (Life Technologies, Carlsbad, CA) according to the manufacturer's instructions. Template cDNA were added to a reaction mixture containing $10 \mu \mathrm{l}$ of $2 \times$ TaqMan Fast Advanced Master Mix (Life Technologies) and endonuclease free water to $20 \mu \mathrm{l}$ and loaded in
TaqMan Array Standard 96 well Plates (Applied Biosystems, Foster City, CA). These plates contain pre-spotted individual TaqMan Gene Expression probes for the detection of genes of interest as well as the house keeping genes $18 \mathrm{~S}, \beta$-actin (ACTB), and GAPDH (Supplementary Table S1). PCR amplification was carried out on a 7900HT Fast Real-Time PCR System (Applied Biosystems) with the following conditions: $95^{\circ} \mathrm{C}, 20 \mathrm{~min} ; 40$ cycles $\left(95^{\circ} \mathrm{C}, 1 \mathrm{~min}\right) ; 20 \mathrm{~min}$ at $60^{\circ} \mathrm{C}$. The 7500 Software v2.0.6 (Applied Biosystems) was used to determine the cycle threshold $(\mathrm{Ct})$ for each reaction and derive the expression ratios relative to control. Wound healing pathway analysis was performed with a RT2 Profiler PCR Mouse Wound Healing Array (Qiagen, Manchester, UK) under the same conditions described above.

Caco2 wound healing assay. The $\mathrm{Caco} 2$ wound healing assay was performed using a modified method. ${ }^{15}$ Briefly, the cells were seeded and grown to confluence in 6 well plates (Thermo Scientific Nunc Cell-Culture Treated, Roskilde, Denmark). The culture medium was discarded, two $0.5-1.0 \mathrm{~mm}$ across linear wounds were made per well with a $200 \mu \mathrm{L}$ sterile beveled pipette tip (USA Scientific, Enfield, $\mathrm{CT})$ and cells were washed with PBS. PBS, CTBp $(0.3-3 \mu \mathrm{M})$, TGF $\beta 1$ $(0.2 \mathrm{nM})$, and/or an anti-TGF $\beta 1,2,3$ antibody (3.85 nM; Abcam, Cambridge, MA) were subsequently added in fresh serum-deprived medium. Photomicrographs of the wounds were taken 0,24 , and $48 \mathrm{~h}$ after the wounding at $\times 4$ magnification. Quantification of the remaining cell-free area to the initial wound area was measured using the public domain software Image J (http://rsbweb.nih.gov), and calculated as a mean percentage per well. The culture medium/ supernatants were collected from each well $48 \mathrm{~h}$ after wounding and stored $-80{ }^{\circ} \mathrm{C}$ until analysis. The culture supernatants were analyzed by a human Cytokine/Chemokine or TGF $\beta 1,2,3$ Magnetic Bead Panel (EMD Millipore, St. Charles, MO). The panel was analyzed with a Milliplex MAP Kit on a MagPix with Luminex xMAP technology.

Immunohistochemistry. Colons were removed and washed with PBS. A portion of the distal colon was fixed with paraformaldehyde overnight and stored in $70 \%$ ethanol until paraffin embedding and sectioning. Sections were deparaffinized with Citrisolv and rehydrated through several ethanol washing steps ending with incubation in distilled water. Antigen retrieval was performed overnight with a 2100 Retriever (Electron Microscopy Sciences) using a pH 8.0 buffer. Tissue sections were blocked for endogenous peroxidase, avidin, biotin, and serum from the animal in which the secondary antibody was raised. Primary antibody (anti-F4/80; Abcam) was incubated with the tissue sections for $2 \mathrm{~h}$ at room temperature. The Vectastain Elite ABC kit (rabbit anti-goat; Vector Labs, Berlingame, CA) was used to label the primary antibody. F4/80 + cells were visualized with the ImmPACT DAB Substrate Kit (Vector Labs) and then dehydrated through an ethanol gradient and finally incubated with Citrisolv. Sections were scanned using a Aperio ScanScope CS (Leica Biosystems, Buffalo Grove, IL) and positive cells were counted, in a blinded manner, in 10 representative sections ( $\times 40$ magnification) from each colon. The 10 sections were averaged and that was the score for each animal.

Histology. Colons were removed and washed with PBS. A portion of the distal colon was fixed with paraformaldehyde overnight and stored in $70 \%$ ethanol until paraffin embedding, sectioning and routine $\mathrm{H} \& \mathrm{E}$ staining. Inflammation scoring was performed as previously described. ${ }^{25}$ Tissue sections from 8 mice were scored in a blinded manner and averaged for each group. Masson's Trichrome Stain was performed using a kit purchased from Electron Microscopy Sciences (Masson's Trichrome for Connective Tissues).

Protein isolation and quantification. Distal colon tissue isolated at sacrifice was snap frozen in liquid nitrogen and pulverized with a Bessman Tissue Pulverizer and placed in T-PER (Thermo Scientific) with a protease inhibitor cocktail (Sigma-Aldrich, St. Louis, MO). Total protein was isolated by gravity centrifugation of tissue fragments 


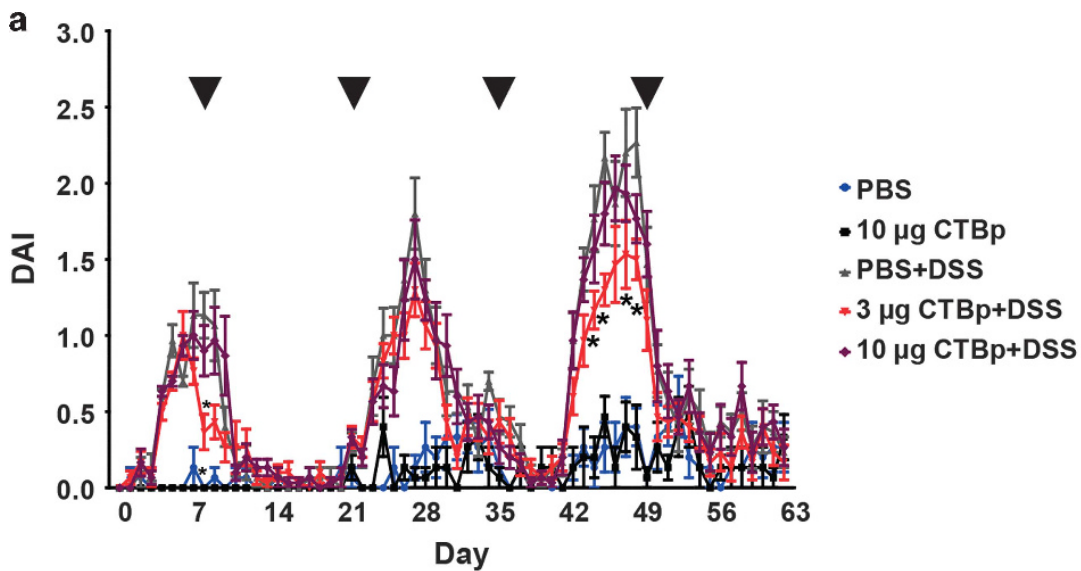

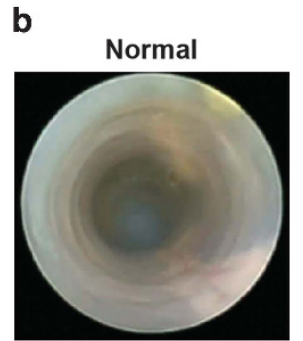

Grade 3
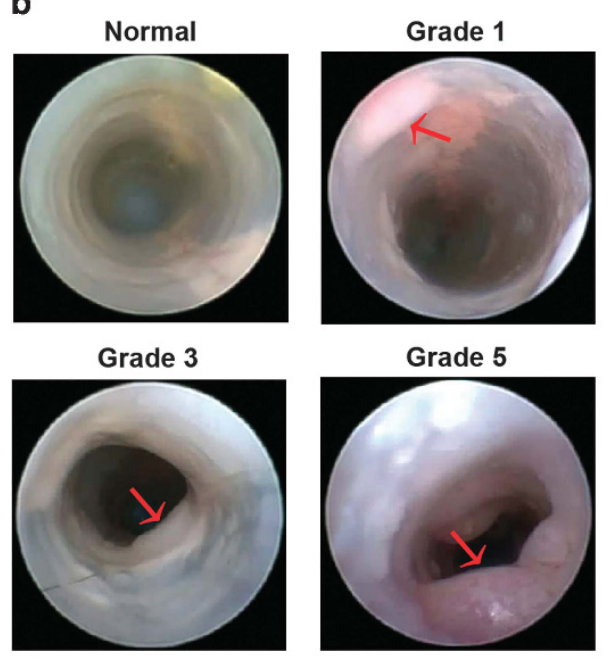
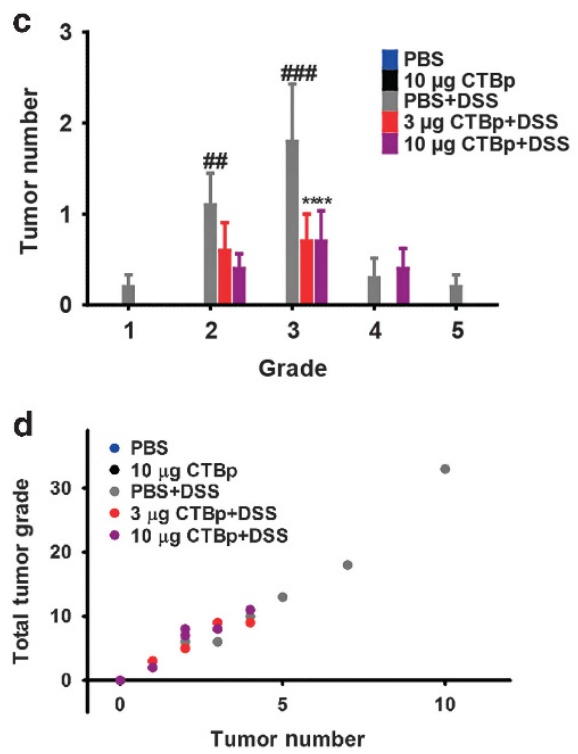
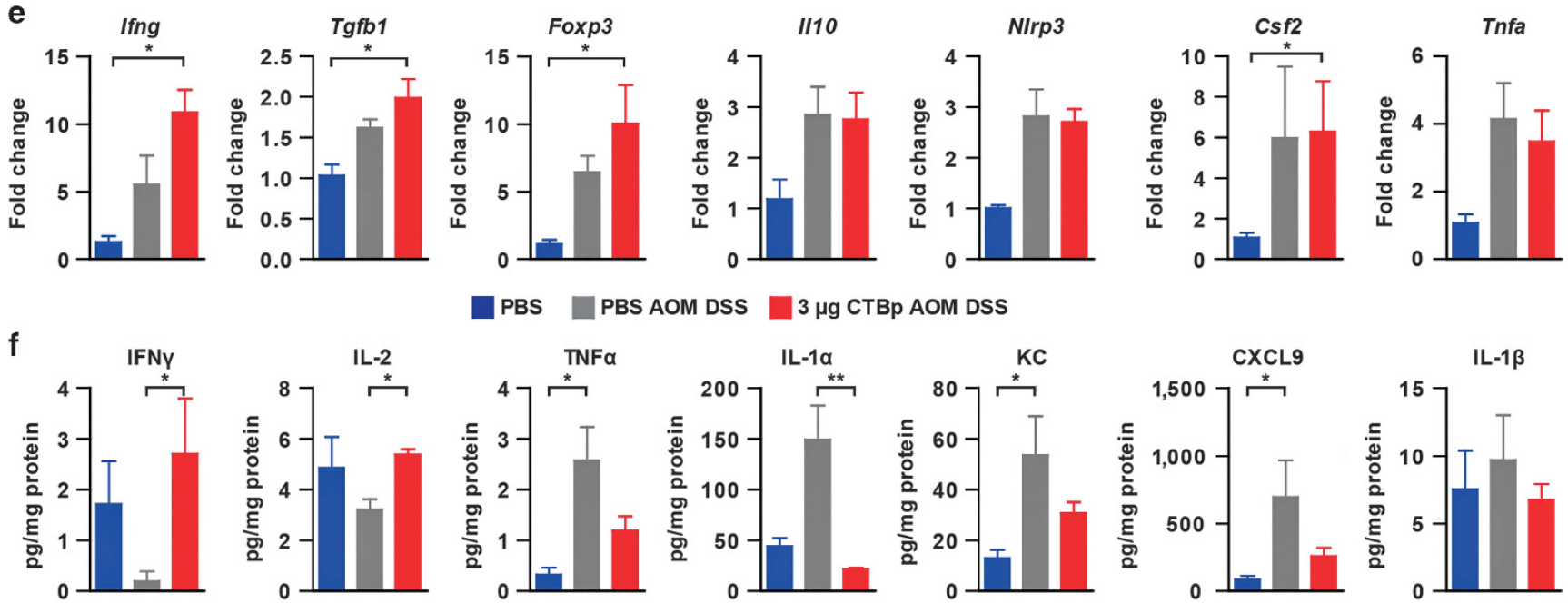

PBS PBS AOM DSS $3 \mu \mathrm{g}$ СTBp AOM DSS
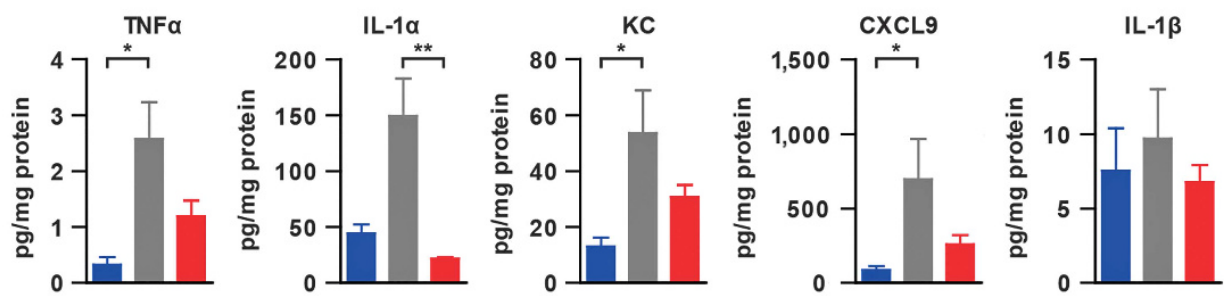

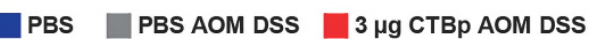

followed by collection of the buffer containing isolated protein, and storage at $-80{ }^{\circ} \mathrm{C}$ until analysis. Protein concentrations were determined and normalized for all samples prior to loading on a Mouse
Cytokine/Chemokine Magnetic Bead Panel (EMD Millipore). The panel was analyzed with a Milliplex MAP Kit on a MagPix with Luminex xMAP technology. 
Figure 7 Biweekly oral therapy with CTBp can mitigate chronic colitis and colitis-associated tumorigenesis. Mice (female C57BL/6 J, 8-week old) received azoxymethane (AOM) i.p. one week prior to DSS exposure. Mice were given water or 2\% DSS water for 7 days followed by water for two weeks; the cycle was repeated two additional times. Mice were dosed orally with PBS, $3 \mu \mathrm{g}$ CTBp or $10 \mu \mathrm{g}$ CTBp on days 7, 21, 35, and 49 (black arrows). (a) DAI. Body weights, fecal consistency and occult blood were scored daily. ${ }^{*} P<0.05$ compared with PBS + DSS group; a repeated-measures ANOVA with Bonferroni Correction. (b) Representative tumor scoring. Tumors were scored from 0 to 5 with 0 being normal tissue and 5 being greater than $50 \%$ of colon circumference tumor invasion. Red arrows indicate representative tumors. (c) Tumor scoring results. Tumors were scored based on the scale in b. ${ }^{\# \#} P<0.01$ and ${ }^{\# \# \#} P<0.001$ compared with PBS and ${ }^{* *} P<0.01$ compared with PBS + DSS; a repeated-measures ANOVA with Bonferroni Correction. (d) Total tumor grade vs. total tumor number. A dot represents each mouse. Mice with no tumors are at the axis intersection. Mean $\pm \mathrm{s}$.e.m. is shown $(n=5$ for PBS group and all other groups $n=10$ ). (e) qRT-PCR analysis of cytokine gene expression in mouse colon tissue. Mean \pm s.e.m. is shown for each group $(N=5)$. (f) Cytokine concentrations in colon tissue lysate. $N=5$ per group. For $\mathbf{e}$ and f , a Kruskal-Wallis test with Dunn's multiple-comparison post-test was used to compare all pairs of groups. Significantly different pairs are highlighted with asterisks $\left({ }^{\star} P<0.05\right.$ and $\left.{ }^{\star \star} P<0.01\right)$.

Tumor scoring. Tumors were scored via endoscopic analysis of the full length of the colon. Tumor scoring was based on the following rubric: 0 for no tumor, 1 is a very small but detectable tumor, 2 the tumor covers up to $1 / 8$ colon circumference, 3 tumor covers $1 / 4$ of colon circumference, 4 tumor covers up to $1 / 2$ of colon, and 5 tumor covers more than $1 / 2$ of colon (Figure $7 \mathbf{b}$ ).

Statistics. For all data, outliers were determined by statistical analysis using the Grubb's test $(P<0.05)$ and excluded from further analysis. Graphs were prepared and analyzed using Graphpad Prism version 5.0 (Graphpad Software, La Jolla, CA). To compare two data sets, an unpaired, two-tailed Student's $t$ test was used. To compare three or more data sets, one-way ANOVA with Bonferroni's multiple-comparison post-test or Kruskal-Wallis test with Dunn's multiplecomparison post-test were performed. For body weights and DAI results, a two-way ANOVA with Bonferroni's multiple-comparison post-test was employed.

SUPPLEMENTARY MATERIAL is linked to the online version of the paper at http://www.nature.com/mi

\section{ACKNOWLEDGMENTS}

We would like to thank Stephen P. Slone for his help in developing the inflammation scoring rubric. We are grateful to Amanda Lasnik and Lauren Moore for technical assistance, and Adam Husk and Kenneth Palmer for critical reading of the manuscript. This work was supported by DoD/ USMRAATTATRC/ W81XWH-10-2-0082-CLIN2 and the Helmsley Charitable Trust Fund. K.J.B. was supported by a T32 Environmental Health Sciences Grant (3 T32 ES 11564-10 S1).

\section{AUTHOR CONTRIBUTIONS}

K.J.B., J.M.R. and N.M. conceived and designed experiments, analyzed data and wrote the manuscript. K.J.B. and J.M.R. performed experiments and contributed equally to the work. K.Y. assisted flow cytometry analysis. K.T.H. produced CTBp and G33D-CTBp. G.W.D. performed endoscopic evaluation of tumors. B.H., V.R.J. and J.C.K provided technical support and edited the manuscript. N.M. supervised the work.

\section{DISCLOSURE}

K.J.B., N.M. and J.M.R. have filed for a provisional patent regarding CTBp's mucosal wound healing activity (U.S. provisional patent application serial no. $62 / 186,151)$.

c 2017 Society for Mucosal Immunology

\section{REFERENCES}

1. Sanchez, J. \& Holmgren, J. Cholera toxin structure, gene regulation and pathophysiological and immunological aspects. Cell Mol. Life Sci. 65, 1347-1360 (2008).

2. Clemens, J., Shin, S., Sur, D., Nair, G.B. \& Holmgren, J. New-generation vaccines against cholera. Nat. Rev. Gastroenterol. Hepatol. 8, 701-710 (2011).
3. Baldauf, K.J., Royal, J.M., Hamorsky, K.T. \& Matoba, N. Cholera toxin B: one subunit with many pharmaceutical applications. Toxins (Basel) 7 , 974-996 (2015).

4. Sun, J.B., Czerkinsky, C. \& Holmgren, J. Mucosally induced immunological tolerance, regulatory $T$ cells and the adjuvant effect by cholera toxin $B$ subunit. Scand. J. Immunol. 71, 1-11 (2010).

5. Stanford, M. et al. Oral tolerization with peptide 336-351 linked to cholera toxin B subunit in preventing relapses of uveitis in Behcet's disease. Clin. Exp. Immunol. 137, 201-208 (2004).

6. Coccia, E.M. et al. Cholera toxin subunit B inhibits IL-12 and IFN-\{gamma\} production and signaling in experimental colitis and Crohn's disease. Gut 54, 1558-1564 (2005).

7. Stal, P. et al. Clinical trial: the safety and short-term efficacy of recombinant cholera toxin B subunit in the treatment of active Crohn's disease. Aliment Pharmacol. Ther. 31, 387-395 (2010).

8. Hamorsky, K.T. et al. Rapid and scalable plant-based production of a cholera toxin $B$ subunit variant to aid in mass vaccination against cholera outbreaks. PLoS Negl. Trop. Dis. 7, e2046 (2013).

9. Hamorsky, K.T. et al. N-glycosylation of cholera toxin B subunit in Nicotiana benthamiana: impacts on host stress response, production yield and vaccine potential. Sci. Rep. 5, 8003 (2015).

10. Vaughn, B.P., Shah, S. \& Cheifetz, A.S. The role of mucosal healing in the treatment of patients with inflammatory bowel disease. Curr. Treat Options Gastroenterol. 12, 103-117 (2014).

11. Anhe, F.F. et al. A polyphenol-rich cranberry extract protects from diet-induced obesity, insulin resistance and intestinal inflammation in association with increased Akkermansia spp. population in the gut microbiota of mice. Gut 64, 872-883 (2015).

12. Gutierrez-Orozco, F. et al. Intestinal microbial dysbiosis and colonic epithelial cell hyperproliferation by dietary alpha-mangostin is independent of mouse strain. Nutrients 7, 764-784 (2015).

13. Balogh, P., Katz, S. \& Kiss, A.L. The role of endocytic pathways in TGF-beta signaling. Pathol. Oncol. Res. 19, 141-148 (2013).

14. Beck, P.L., Rosenberg, I.M., Xavier, R.J., Koh, T., Wong, J.F. \& Podolsky, D.K. Transforming growth factor-beta mediates intestinal healing and susceptibility to injury in vitro and in vivo through epithelial cells. Am. J. Pathol. 162, 597-608 (2003).

15. Bulut, K. et al. Glucagon-like peptide 2 improves intestinal wound healing through induction of epithelial cell migration in vitroevidence for a TGF-beta-mediated effect. Regul. Pept. 121, 137-143 (2004).

16. Jobling, M.G. \& Holmes, R.K. Analysis of structure and function of the B subunit of cholera toxin by the use of site-directed mutagenesis. Mol. Microbiol. 5, 1755-1767 (1991).

17. Jobling, M.G. \& Holmes, R.K. Mutational analysis of ganglioside GM(1)binding ability, pentamer formation, and epitopes of cholera toxin B (CTB) subunits and $\mathrm{CTB} /$ heat-labile enterotoxin B subunit chimeras. Infect. Immun. 70, 1260-1271 (2002).

18. Jones-Hall, Y.L. \& Grisham, M.B. Immunopathological characterization of selected mouse models of inflammatory bowel disease: Comparison to human disease. Pathophysiology 21, 267-288 (2014).

19. Wynn, T.A. \& Vannella, K.M. Macrophages in Tissue Repair, Regeneration, and Fibrosis. Immunity 44, 450-462 (2016).

20. Principi, M. et al. Fibrogenesis and fibrosis in inflammatory bowel diseases: Good and bad side of same coin?. World J. Gastrointest. Pathophysiol. 4, 100-107 (2013). 
21. Cutroneo, K.R. TGF-beta-induced fibrosis and SMAD signaling: oligo decoys as natural therapeutics for inhibition of tissue fibrosis and scarring. Wound Repair Regen. 15 (Suppl 1), S54-S60 (2007).

22. Desreumaux, P. \& Ghosh, S. Review article: mode of action and delivery of 5-aminosalicylic acid - new evidence. Aliment Pharmacol. Ther. 24 (Suppl 1), 2-9 (2006).

23. Sun, J.B., Czerkinsky, C. \& Holmgren, J. B lymphocytes treated in vitro with antigen coupled to cholera toxin B subunit induce antigen-specific Foxp3(+) regulatory Tcells and protect against experimental autoimmune encephalomyelitis. J. Immunol. 188, 1686-1697 (2012).

24. Ma, D., Wolvers, D., Stanisz, A.M. \& Bienenstock, J. Interleukin-10 and nerve growth factor have reciprocal upregulatory effects on intestinal epithelial cells. Am. J. Physiol. Regul. Integr. Comp. Physiol. 284, R1323-R1329 (2003).

25. Cooper, H.S., Murthy, S.N., Shah, R.S. \& Sedergran, D.J. Clinicopathologic study of dextran sulfate sodium experimental murine colitis. Lab Invest. 69, 238-249 (1993).

26. Wilgus, T.A., Roy, S. \& McDaniel, J.C. Neutrophils and Wound Repair: Positive Actions and Negative Reactions. Adv. Wound Care (New Rochelle) 2, 379-388 (2013).

27. Leoni, G., Neumann, P.A., Sumagin, R., Denning, T.L. \& Nusrat, A. Wound repair: role of immune-epithelial interactions. Mucosal. Immunol. 8, 959-968 (2015).

28. Taverna, D., Pollins, A.C., Sindona, G., Caprioli, R.M. \& Nanney, L.B. Imaging mass spectrometry for assessing cutaneous wound healing: analysis of pressure ulcers. J. Proteome Res. 14, 986-996 (2015).

29. Neurath, M.F. New targets for mucosal healing and therapy in inflammatory bowel diseases. Mucosal. Immunol. 7, 6-19 (2014).

30. Scarpa, M. et al. The epithelial danger signal IL-1alpha is a potent activator of fibroblasts and reactivator of intestinal inflammation. Am. J. Pathol. 185, 1624-1637 (2015).

31. Wang, L., Wang, Y., Song, Z., Chu, J. \& Qu, X. Deficiency of interferongamma or its receptor promotes colorectal cancer development. J. Interferon Cytokine Res. 35, 273-280 (2015).

32. Grande, C., Firvida, J.L., Navas, V. \& Casal, J. Interleukin-2 for the treatment of solid tumors other than melanoma and renal cell carcinoma. Anticancer Drugs 17, 1-12 (2006).

33. Kim, P.H., Eckmann, L., Lee, W.J., Han, W. \& Kagnoff, M.F. Cholera toxin and cholera toxin $B$ subunit induce IgA switching through the action of TGF-beta 1. J. Immunol. 160, 1198-1203 (1998).

34. Walsh, A., Palmer, R. \& Travis, S. Mucosal healing as a target of therapy for colonic inflammatory bowel disease and methods to score disease activity. Gastrointest. Endosc. Clin. N. Am. 24, 367-378 (2014).
35. Satoh, Y. et al. Cyclosporine regulates intestinal epithelial apoptosis via TGF-beta-related signaling. Am. J. Physiol. Gastrointest Liver Physiol. 297, G514-G519 (2009).

36. Itokazu, Y., Pagano, R.E., Schroeder, A.S., O'Grady, S.M., Limper, A.H. \& Marks, D.L. Reduced GM1 ganglioside in CFTR-deficient human airway cells results in decreased beta1-integrin signaling and delayed wound repair. Am. J. Physiol. Cell Physiol. 306, C819-C830 (2014).

37. Biancheri, P., Giuffrida, P., Docena, G.H., MacDonald, T.T., Corazza, G.R. \& Di Sabatino, A. The role of transforming growth factor (TGF)-beta in modulating the immune response and fibrogenesis in the gut. Cytokine Growth Factor Rev. 25, 45-55 (2014).

38. Oshima, H. et al. Suppressing TGFbeta signaling in regenerating epithelia in an inflammatory microenvironment is sufficient to cause invasive intestinal cancer. Cancer Res. 75, 766-776 (2015).

39. Calabrese, E.J. \& Baldwin, L.A. The hormetic dose-response model is more common than the threshold model in toxicology. Toxicol. Sci. 71, 246-250 (2003).

40. Calabrese, E.J. Historical foundations of wound healing and its potential for acceleration: dose-response considerations. Wound Repair Regen. 21, 180-193 (2013).

41. van Deventer, S.J., Elson, C.O. \& Fedorak, R.N. Multiple doses of intravenous interleukin 10 in steroid-refractory Crohn's disease. Crohn's Disease Study Group. Gastroenterology 113, 383-389 (1997).

42. Targan, S.R. et al. A short-term study of chimeric monoclonal antibody cA2 to tumor necrosis factor alpha for Crohn's disease. Crohn's Disease cA2 Study Group. N. Engl. J. Med. 337, 1029-1035 (1997).

43. Barker, N. Adult intestinal stem cells: critical drivers of epithelial homeostasis and regeneration. Nat. Rev. Mol. Cell Biol. 15, 19-33 (2014).

44. Lysakova-Devine, T., O'Farrelly, C. \& Tissue-specific, N.K. cell populations and their origin. J. Leukoc. Biol. 96, 981-990 (2014).

45. Kiesler, P., Fuss, I.J. \& Strober, W. Experimental models of inflammatory bowel diseases. Cell Mol. Gastroenterol. Hepatol. 1, 154-170 (2015).

46. Wirtz, S., Neufert, C., Weigmann, B. \& Neurath, M.F. Chemically induced mouse models of intestinal inflammation. Nat. Protoc. 2, 541-546 (2007).

47. Lefrancois, L. \& Lycke, N. Isolation of mouse small intestinal intraepithelial lymphocytes, Peyer's patch, and lamina propria cells. Curr. Protoc. Immunol. Chapter 3, Unit 319 (2001).

48. Scheiffele, F. \& Fuss, I.J. Induction of TNBS colitis in mice. Curr. Protoc. Immunol. Chapter 15, Unit 1519 (2002).

49. Irizarry, R.A. et al. Exploration, normalization, and summaries of high density oligonucleotide array probe level data. Biostatistics 4, 249-264 (2003). 